\title{
Magnetars: structure and evolution from p-star models
}

\author{
P. Cea ${ }^{1,2}$ \\ 1 Dipartimento Interateneo di Fisica, Università di Bari, via G. Amendola 173, 70126 Bari, Italy \\ e-mail: Paolo.Cea@ba.infn.it \\ 2 INFN - Sezione di Bari, Via G. Amendola 173, 70126 Bari, Italy
}

Received 20 June 2005 / Accepted 22 November 2005

\section{ABSTRACT}

P-stars are compact stars made of up-and-down quarks in $\beta$-equilibrium with electrons in a chromomagnetic condensate. We discuss p-stars endowed with super strong dipolar magnetic field that, following consolidated tradition in literature, are referred to as magnetars. We show that soft gamma-ray repeaters and anomalous X-ray pulsars can be understood within our theory. We find a well-defined criterion to distinguish rotation-powered pulsars from magnetic-powered pulsars. We show that glitches, which in our magnetars are triggered by magnetic dissipative effects in the inner core, explain both the quiescent emission and bursts in soft gamma-ray repeaters and anomalous X-ray pulsars. We account for the braking glitch from SGR 1900+14 and the normal glitch from AXP 1E 2259+586 following a giant burst. We discuss and explain the observed anti correlation between hardness ratio and intensity. Within our magnetar theory we are able to quantitatively account for light curves for both gamma-ray repeaters and anomalous X-ray pulsars. In particular we explain the puzzling light curve after the June 18, 2002 giant burst from AXP 1E 2259+586.

Key words. stars: pulsars: general - stars: pulsars: individual: SGR 1900+14 - pulsars: individual: AXP 1E 2259+586

\section{Introduction}

In a few years since their discovery (Hewish et al. 1968), pulsars have been identified with rotating neutron stars, first predicted theoretically by Baade \& Zwicky (1934a,b,c), endowed with a strong magnetic field (Pacini 1968; Gold 1968). Nowadays, no one doubts that pulsars are neutron stars, even though it should be remembered that there may be alternative explanations for them.

Up to the present it seems that there are no alternative models able to provide as satisfactory an explanation for the wide variety of pulsar phenomena as those built around the rotating neutron star model. However, quite recently we proposed (Cea 2004a,b) a new class of compact stars, named p-stars, made of up-and-down quarks in $\beta$-equilibrium with electrons in an Abelian chromomagnetic condensate that is challenging the standard model based on neutron stars.

In the present paper we investigate the properties of anomalous X-ray pulsars (AXPs) and soft gamma-ray repeaters (SGRs). For a recent review of the observational properties of anomalous X-ray pulsars see Mereghetti (1999), Mereghetti et al. (2002), and Kaspi \& Gavriil (2004), while for soft gamma-ray repeaters see Hurley (1999) and Woods (2003). Recently, these two groups have been linked by the discovery of persistent emission from soft gamma ray repeaters that is very similar to anomalous X-ray pulsars and of a bursting activity in anomalous X-ray pulsars that is quite similar to soft gamma ray repeaters (see, for instance, Kaspi 2004; Woods \& Thompson 2004). Duncan \& Thompson (1992) and Paczyński (1992) have proposed that soft gamma-ray repeaters are pulsars whose surface magnetic fields exceed the critical magnetic field $B_{\mathrm{QED}} \simeq 4.4 \times 10^{13}$ Gauss. Indeed, Duncan \& Thompson (1992) refer to these pulsar as magnetars. In particular Duncan \& Thompson $(1995,1996)$ argue that the soft gamma-ray repeater bursts and quiescent emission were powered by the decay of an ultra-high magnetic field. This interpretation is based on observations that showed that these peculiar pulsars are slowing down rapidly, with an inferred magnetic dipole field much greater than the quantum critical field $B_{\mathrm{QED}}$, while producing steady emission at a rate far in excess of the rotational kinetic energy loss.

The main purpose of this paper is to discuss in detail pstars endowed with a super strong dipolar magnetic field which, following a well-consolidated tradition in the literature, will be referred to as magnetars. We will show that soft gammaray repeaters and anomalous X-ray pulsars can be understood within our theory. The plan of the paper is as follows. In Sect. 2 we discuss the phenomenological evidence for the dependence of pulsar magnetic fields on the rotational period. We argue that there is a well-defined criterion that allows us to distinguish between rotation powered pulsars and magnetic powered 
pulsars. We explicitly explain why the recently discovered high magnetic field radio pulsars are indeed rotation powered pulsars. In Sect. 3 we introduce the radio death line, which in the $\dot{P}-P$ plane separated radio pulsars from radio quiet magnetic powered pulsars, and compare it with available observational data. Section 4 is devoted to the glitch mechanism in our magnetars and their observational signatures. In Sect. 4.1 we compare glitches in SGR 1900+14 and 1E 2259+586, our prototypes for a soft gamma-ray repeater and an anomalous X-ray pulsar, respectively. Sections 4.2 and 4.3 are devoted to explaining the origin of the quiescent luminosity, the burst activity, and the emission spectrum during bursts. In Sect. 4.4 we discuss the anti correlation between hardness ratio and intensity. In Sect. 5 we develop a general formalism to cope with light curves for both giant and intermediate bursts. In Sects. 5.1 through 5.4 we carefully compare our theory with the available light curves in literature. In particular, we are able to account for the peculiar light curve following the June 18, 2002 giant burst from the anomalous X-ray pulsar 1E 2259+586. Finally, we draw our conclusions in Sect. 6.

\section{Rotation versus magnetic powered pulsars}

As discussed in Cea (2004a) the structure of p-stars is determined once the equation of state appropriate to the description of deconfined quarks and gluons in a chromomagnetic condensate is specified. In general, the quark chemical potentials are smaller than the strength of the chromomagnetic condensate so that, up-and-down quarks occupy the lowest Landau levels. However, for certain values of the central energy density it happens that a fraction of down quarks must jump into higher Landau levels in the stellar core, leading to a central core with energy density $\varepsilon_{\mathrm{c}}$ that is somewhat greater than the energy density outside the core.

These quarks in the inner core produce a vector current in response to the chromomagnetic condensate. Obviously, the quark current tends to screen the chromomagnetic condensate by a very tiny amount. However, since the down quark has an electric charge $q_{\mathrm{d}}=-\frac{1}{3} e$ ( $e$ is the electric charge), the quark current generates a uniform magnetic field in the core parallel to the chromomagnetic condensate with strength $B_{\mathrm{c}} \simeq \frac{e}{96 \pi} g H$ (here and in the following we shall adopt natural units $\hbar=c=k_{B}=1$ ). The inner core is characterized by huge conductivity, while outer core quarks are frozen into the lowest Landau levels. Due to the energy gap between the lowest Landau levels and the higher ones, the quarks outside the core cannot support any electrical current. As a consequence, the magnetic field in the region outside the core is dipolar, leading to the surface magnetic field:

$B_{\mathrm{S}} \simeq B_{\mathrm{c}}\left(\frac{R_{\mathrm{c}}}{R}\right)^{3}, \quad B_{\mathrm{c}} \simeq \frac{e}{96 \pi} g H$,

$R$ and $R_{\mathrm{c}}$ being the stellar and inner core radii, respectively. In general the formation of an inner core that is denser than the outer core is obstructed by the centrifugal force produced by stellar rotation. Since the centrifugal force is proportional to the square of the stellar rotation frequency, this leads us to

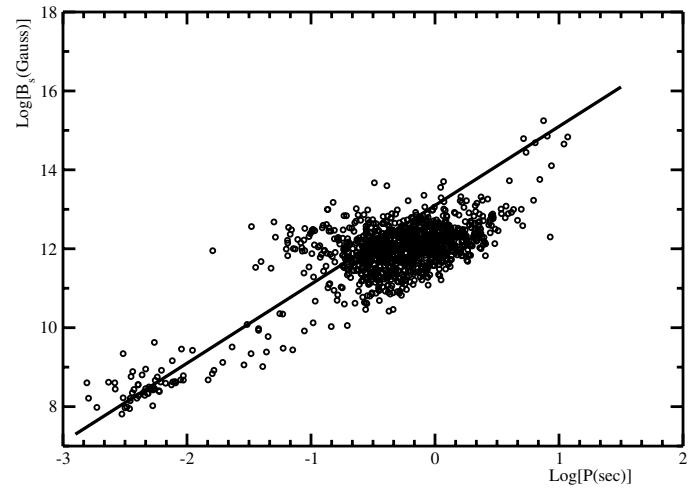

Fig. 1. Inferred magnetic field $B_{\mathrm{S}}$ plotted versus stellar period for 1194 pulsars taken from the ATNF Pulsar Catalog (www.atnf.csiro.au/research/pulsar/psrcat). The full line corresponds to Eq. (2) with $B_{1} \simeq 1.3 \times 10^{13}$ Gauss.

argue that the surface magnetic field strength is proportional to the square of the stellar period:

$B_{\mathrm{S}} \simeq B_{1}\left(\frac{P}{1 \mathrm{~s}}\right)^{2}$,

where $B_{1}$ is the surface magnetic field for pulsars with nominal period $P=1 \mathrm{~s}$. Indeed, in Fig. 1 we display the surface magnetic field strength $B_{\mathrm{S}}$ inferred from (for instance, see Manchester \& Taylor 1977):

$B_{\mathrm{S}} \simeq 3.1 \times 10^{19} \sqrt{P \dot{P}}$ Gauss,

versus the period. Remarkably, by assuming $B_{1} \simeq 1.3 \times$ $10^{13}$ Gauss, we find that Eq. (2) accounts rather well for the inferred magnetic field for pulsars ranging from millisecond pulsars up to anomalous X-ray pulsars and soft-gamma repeaters. As a consequence of Eq. (2), we see that the dipolar magnetic field is time dependent. In fact, it is easy to find

$B_{\mathrm{S}}(t) \simeq B_{0} \quad\left(1+2 \frac{\dot{P}}{P} t\right)$,

where $B_{0}$ indicates the magnetic field at the initial observation time. Note that Eq. (4) implies that the magnetic field varies on a time scale given by the characteristic age.

It is widely accepted that pulsar radio emission is powered by the rotational energy

$E_{\mathrm{R}}=\frac{1}{2} I \omega^{2}$,

so that the spin-down power output is given by:

$-\dot{E}_{\mathrm{R}}=-I \omega \dot{\omega}=4 \pi^{2} I \frac{\dot{P}}{P^{3}}$.

On the other hand, an important source of energy is provided by the magnetic field. In fact, the classical energy stored into the magnetic field is:

$E_{B}=\frac{1}{8 \pi} \int_{r \geq R} \mathrm{~d}^{3} r B^{2}(r)$.

Assuming a dipolar magnetic field:

$B(r)=B_{\mathrm{S}}\left(\frac{R}{r}\right)^{3}$ for $r \geq R$, 
Equation (7) leads to:

$E_{B}=\frac{1}{6} B_{\mathrm{S}}^{2} R^{3}$.

Now, from Eq. (4) the surface magnetic field is time dependent, so that the magnetic power output is given by:

$\dot{E}_{B}=\frac{2}{3} B_{0}^{2} R^{3} \quad \frac{\dot{P}}{P}$.

For rotation-powered pulsars it turns out that $\dot{E}_{B} \ll-\dot{E}_{\mathrm{R}}$. However, if the dipolar magnetic field is strong enough, then the magnetic power Eq. (10) can be on the order or even greater than, the spin-down power. Thus, we may formulate a welldefined criterion to distinguish rotation powered pulsars from magnetic powered pulsars. Until $\dot{E}_{B}<-\dot{E}_{\mathrm{R}}$ there is enough rotation power to sustain the pulsar emission. On the other hand, when $\dot{E}_{B} \geq-\dot{E}_{\mathrm{R}}$ all the rotation energy is stored in the increasing magnetic field, and the pulsar emission is turned off. In fact, in the next section we derive the radio death line, which is the line that in the $P-\dot{P}$ plane separates rotation-powered pulsars from magnetic-powered pulsars.

In the remainder of this section, we discuss the recently detected radio pulsars with very strong surface magnetic fields, and focus on the two radio pulsars with the strongest surface magnetic field: PSR J1718-3718 and PSR J1847-0130. These pulsars have inferred surface magnetic fields well above the quantum critical field $B_{\mathrm{QED}}$ above which some models (Baring \& Harding 1998) predict that radio emission should not occur. In particular, we have:

PSR J1718-3718 (Hobbs et al. 2004)

$P \simeq 3.4 \mathrm{~s}, B_{\mathrm{S}} \simeq 7.4 \times 10^{13}$ Gauss,

PSR J1847-0130 (McLaughlin et al. 2003)

$P \simeq 6.7 \mathrm{~s}, B_{\mathrm{S}} \simeq 9.4 \times 10^{13}$ Gauss.

Both pulsars have average radio luminosities and larger surface magnetic fields than does AXP 1E 2259+586. Now, using Eqs. (10), (6), together with $I=\frac{2}{5} M R^{2}$ and Eq. (11), we get:

PSR J1718-3718

$-\dot{E}_{\mathrm{R}} \simeq 3.4 \times 10^{45} \mathrm{erg} \frac{\dot{P}}{P}, \dot{E}_{B} \simeq 4.7 \times 10^{44} \mathrm{erg} \frac{\dot{P}}{P}$,

PSR J1847-0130

$-\dot{E}_{\mathrm{R}} \simeq 8.8 \times 10^{44} \operatorname{erg} \frac{\dot{P}}{P}, \dot{E}_{B} \simeq 7.5 \times 10^{44} \operatorname{erg} \frac{\dot{P}}{P}$.

We see that in any case $\dot{E}_{B}<-\dot{E}_{R}$, so that there is enough rotational energy to power the pulsar emission.

\section{Radio death line}

As discussed above, until $\dot{E}_{B}<-\dot{E}_{\mathrm{R}}$ the rotation power loss sustains the pulsar emission. We have already shown that this explains the pulsar activity for pulsars with inferred magnetic fields well above the critical field $B_{\mathrm{QED}}$. In this section we explain why anomalous X-ray pulsars and soft gamma repeaters are radio-quiet pulsars.

When $\dot{E}_{B} \geq-\dot{E}_{\mathrm{R}}$, all the rotation energy is stored in the increasing magnetic field, and the pulsar emission is turned

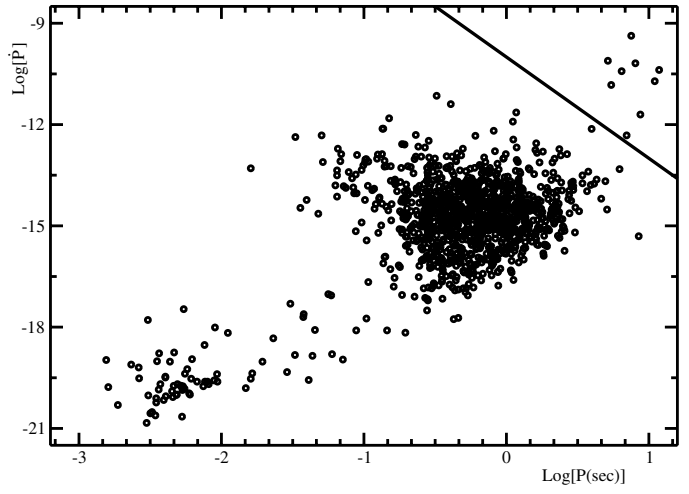

Fig. 2. Period derivative plotted versus stellar period for 1194 pulsars taken from the ATNF Pulsar Catalog (www.atnf.csiro.au/ research/pulsar/psrcat). The full line corresponds to Eq. (16).

off. As a consequence pulsars with sufficiently strong magnetic fields are radio quiet. Accordingly we see that the condition

$\dot{E}_{B}=-\dot{E}_{\mathrm{R}}$

is able to distinguish rotation powered pulsars from magnetic powered pulsars. Now, using (Manchester \& Taylor 1977)

$B_{\mathrm{S}} \simeq \sqrt{\frac{3 I P \dot{P}}{8 \pi^{2} R^{6}}}$,

we recast Eq. (13) as

$P^{3} \dot{P}=16 \pi^{4} R^{3}$.

Using the canonical radius $R \simeq 10 \mathrm{~km}$, we get:

$3 \log (P)+\log (\dot{P}) \simeq-10$.

Equation (16) is a straight line as plotted in Fig. 2 in the $\log (P)-\log (\dot{P})$ plane. In Fig. 2 we also display 1194 pulsars taken from the ATNF Pulsar Catalog (www .atnf.csiro.au/ research/pulsar/psrcat). We see that rotation-powered pulsars, ranging from millisecond pulsars up to radio pulsars, do indeed lie below our Eq. (16). In Fig. 2 the recently detected high magnetic field pulsars are not included. However, we have already argued in the previous section that these pulsars have spin parameters that indicate that these pulsars are rotation powered. On the other hand, Fig. 2 shows that all soft gamma-ray repeaters and anomalous X-ray pulsars in the ATNF Pulsar Catalog lie above our line Eq. (16). In particular, in Fig. 2 the pulsar above and nearest to the line Eq. (16) corresponds to AXP 1E 2259+586. From this we see that our radio dead line, Eq. (16), correctly predicts that AXP 1E 2259+586 is not a radio pulsar even though the magnetic field is lower than in radio pulsars PSR J1718-3718 and PSR J1847-0130.

We may conclude that pulsars above our dead line are magnetars, i.e. magnetic-powered pulsars. The emission properties of magnetars are quite different from rotation-powered pulsars: the emission from magnetars consists in thermal blackbody radiation from the surface. 


\section{Glitches in magnetars}

The origin of the dipolar magnetic field in p-stars is in the inner core being uniformly magnetized. In Fig. 3 we display a schematic view of the interior of a p-star. The presence of the uniformly magnetized inner core leads to a well-defined glitch mechanism in p-stars. Indeed, dissipative effects, which are more pronounced in young stars, tend to decrease the strength of the magnetic field. On the other hand, when $B_{\mathrm{c}}$ decreases due to dissipation effects, then the magnetic flux decreases locally and, according to Lenz's law, induces a current that resists the reduction of the magnetic flux. This means that some quarks must flow into the core by jumping onto higher Landau levels. In other words, the core radius must increase. Moreover, due to the very high conductivity of quarks in the core, we have:

$B_{\mathrm{c}} R_{\mathrm{c}}^{2} \simeq$ const.,

which implies:

$\frac{\delta B_{\mathrm{c}}}{B_{\mathrm{c}}}+2 \frac{\delta R_{\mathrm{c}}}{R_{\mathrm{c}}} \simeq 0$,

or

$\frac{\delta R_{\mathrm{c}}}{R_{\mathrm{c}}} \simeq-\frac{1}{2} \frac{\delta B_{\mathrm{c}}}{B_{\mathrm{c}}}$

Equation (19) confirms that an increase of the inner core radius $\delta R_{\mathrm{c}}>0$ corresponds to the decrease in the core magnetic field, $\delta B_{\mathrm{c}}<0$. This sudden variation of the radius of the inner core leads to glitches. It is straightforward to show that the magnetic moment

$m=B_{\mathrm{S}} R^{3}=B_{\mathrm{c}} R_{\mathrm{c}}^{3}$

where we used Eq. (1), must increase in the glitch. Using Eq. (19), we get:

$\frac{\delta m}{m}=\frac{\delta B_{\mathrm{c}}}{B_{\mathrm{c}}}+3 \frac{\delta R_{\mathrm{c}}}{R_{\mathrm{c}}} \simeq \frac{\delta R_{\mathrm{c}}}{R_{\mathrm{c}}}>0$.

Another interesting consequence of the glitch is that the stellar radius $R$ must decrease, i.e. the star contracts. This is an inevitable result of the increase of the inner core, which is characterized by an energy density $\varepsilon_{\mathrm{c}}$ higher than the outer core density $\varepsilon$. Therefore, the variation in radius is negative: $\delta R<0$ (see Fig. 3).

In the radio pulsar, where the magnetic energy can be neglected, conservation of the mass leads to:

$\frac{\delta R}{R} \simeq-\frac{\varepsilon_{\mathrm{c}}-\varepsilon}{\bar{\varepsilon}}\left(\frac{R_{\mathrm{c}}}{R}\right)^{3} \frac{\delta R_{\mathrm{c}}}{R_{\mathrm{c}}}$,

where $\bar{\varepsilon}$ is the average energy density. In general, we may assume that $\frac{\varepsilon_{\mathrm{c}}-\varepsilon}{\bar{\varepsilon}}$ is a constant of order unity, so that Eq. (22) becomes:

$\frac{\delta R}{R} \simeq-\left(\frac{R_{\mathrm{c}}}{R}\right)^{3} \frac{\delta R_{\mathrm{c}}}{R_{\mathrm{c}}}$

Note that the ratio $\left(\frac{R_{\mathrm{c}}}{R}\right)^{3}$ can be estimated from Eq. (1) once the surface magnetic field is known. We find that, even for magnetars, $\left(\frac{R_{\mathrm{c}}}{R}\right)^{3}$ is about $10^{-2}$ or less. Thus our Eqs. (1) and (23) show that:

$\delta R<0, \quad-\frac{\delta R}{R} \ll \frac{\delta R_{\mathrm{c}}}{R_{\mathrm{c}}}$

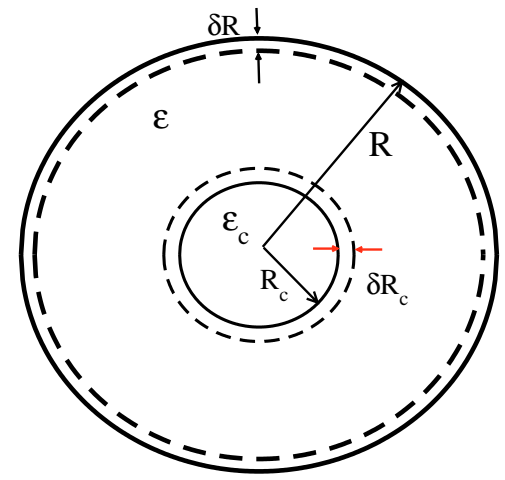

Fig. 3. Schematic view of the interior of a p-star. $R_{\mathrm{c}}$ and $R$ represent the inner core and stellar radii, respectively; $\varepsilon_{\mathrm{c}}$ is the energy density of the inner core, and $\varepsilon$ is the energy density outside the core.

As is well known, because of the external magnetic braking torque, pulsars slow down according to (e.g. see Manchester \& Taylor 1977):

$-\dot{v} \propto m^{2} I^{-1} v^{3}$

so that:

$\frac{\delta \dot{v}}{\dot{v}}=2 \frac{\delta m}{m}-\frac{\delta I}{I}+3 \frac{\delta v}{v}$.

From the conservation of angular momentum we have:

$\left|\frac{\delta I}{I}\right| \simeq\left|\frac{\delta v}{v}\right|$

Moreover, from observational data it turns out that

$\left|\frac{\delta \dot{v}}{\dot{v}}\right| \gg\left|\frac{\delta v}{v}\right|$,

so that Eq. (26) becomes:

$\frac{\delta \dot{v}}{\dot{v}} \simeq 2 \frac{\delta m}{m} \simeq 2 \frac{\delta R_{\mathrm{c}}}{R_{\mathrm{c}}} \simeq-\frac{\delta B_{\mathrm{c}}}{B_{\mathrm{c}}}$,

where we used Eqs. (21) and (19). Equation (29) does show that the variation in the radius of the inner core leads to a glitch.

In rotation-powered pulsars, starting from Eq. (22), one can show that $\left|\frac{\delta v}{v}\right| \simeq\left|\frac{\delta R}{R}\right|$. Taking Eq. (24) into account, we recover the phenomenological relation Eq. (28). A full account of glitches in radio pulsars will be presented elsewhere, but glitches in magnetars are considered in the next section, where we show that Eqs. (24) and (28) indeed hold even in magnetars.

The most dramatic effect induced by glitches in magnetars is the release of a huge amount of magnetic energy in the interior of the star and into the magnetosphere. To see this, let us consider the energy stored in the magnetic field in the interior of the magnetar. We have

$E_{B}^{\mathrm{int}}=\frac{1}{6} R_{\mathrm{c}}^{3} B_{\mathrm{c}}^{2}+\frac{1}{8 \pi} \int_{R_{\mathrm{c}}}^{R} \mathrm{~d}^{3} r\left[B_{\mathrm{c}}\left(\frac{R_{\mathrm{c}}}{r}\right)^{3}\right]^{2}$,

where the first term is the energy stored in the core where the magnetic field is uniform. The variation in the magnetic energy 
Eq. (30) caused by a glitch is easily evaluated. Taking into account both Eq. (19) and $\left(\frac{R_{\mathrm{c}}}{R}\right)^{3} \ll 1$, we get:

$$
\begin{aligned}
\delta E_{B}^{\text {int }} & \simeq-\frac{1}{3} R^{3} B_{\mathrm{S}}^{2}\left(\frac{R}{R_{\mathrm{c}}}\right)^{3} \frac{\delta R_{\mathrm{c}}}{R_{\mathrm{c}}}-\frac{1}{2} R^{3} B_{\mathrm{S}}^{2} \frac{\delta R_{\mathrm{c}}}{R_{\mathrm{c}}} \\
& \simeq-\frac{1}{3} R^{3} B_{\mathrm{S}}^{2}\left(\frac{R}{R_{\mathrm{c}}}\right)^{3} \frac{\delta R_{\mathrm{c}}}{R_{\mathrm{c}}} .
\end{aligned}
$$

Equation (31) shows that there is a decrease in the magnetic energy, so that after a glitch in magnetars a huge magnetic energy is released into the interior of the star. We shall see that this energy is enough to sustain the quiescent emission. On the other hand, the glitch also induces a sudden variation in the magnetic energy stored in the magnetosphere. Indeed, from Eq. (1) we find:

$$
\frac{\delta B_{\mathrm{S}}}{B_{\mathrm{S}}}=\frac{\delta B_{\mathrm{c}}}{B_{\mathrm{c}}}+3 \frac{\delta R_{\mathrm{c}}}{R_{\mathrm{c}}}-3 \frac{\delta R}{R} \simeq \frac{\delta R_{\mathrm{c}}}{R_{\mathrm{c}}}-3 \frac{\delta R}{R} \simeq \frac{\delta R_{\mathrm{c}}}{R_{\mathrm{c}}}>0 .
$$

Thus, the magnetic energy stored in the magnetosphere,

$E_{B}^{\mathrm{ext}}=\frac{1}{8 \pi} \int_{R}^{\infty} \mathrm{d}^{3} r\left[B_{\mathrm{S}}\left(\frac{R}{r}\right)^{3}\right]^{2}=\frac{1}{6} B_{\mathrm{S}}^{2} R^{3}$,

increases by:

$\delta E_{B}^{\mathrm{ext}}=\frac{1}{3} R^{3} B_{\mathrm{S}}^{2}\left(\frac{\delta B_{\mathrm{S}}}{B_{\mathrm{S}}}+\frac{3}{2} \frac{\delta R}{R}\right) \simeq \frac{1}{3} R^{3} B_{\mathrm{S}}^{2} \frac{\delta B_{\mathrm{S}}}{B_{\mathrm{S}}}>0$.

This magnetic energy is injected directly into the magnetosphere, where it is dissipated by the well-defined physical mechanism discussed in Sect. 4.3 and is responsible for bursts in soft gamma-ray repeaters and anomalous X-ray pulsars.

To summarize, in this section we have found that dissipative phenomena in the inner core of a p-star tend to decrease the strength of the core magnetic field. This, in turn, results in an increase in the radius of the core $\delta R_{\mathrm{c}}>0$ and in a contraction of the surface of the star, $\delta R<0$. We have also shown that the glitch releases an amount of magnetic energy in the interior of the star and injects magnetic energy into the magnetosphere, where it is completely dissipated. Below we argue that these magnetic glitches are responsible for the quiescent emission and bursts in gamma-ray repeaters and anomalous X-ray pulsars.

Interestingly enough, in Cheng et al. (1995) it is shown that SGR events and earthquakes share several distinctive statistical properties, namely: power-law energy distributions, log-symmetric waiting time distributions, strong correlations between waiting times of successive events, and weak correlations between waiting times and intensities. These statistical properties of bursts can be easily understood if bursts originate in the release of a small amount of energy from a reservoir of stored energy. As a matter of fact, the burst activity in our theory is accounted for by the release of a tiny fraction of magnetic energy stored in magnetars. Even for giant bursts we find that the released energy is a few per cent of the magnetic energy. Moreover, Hurley et al. (1994) noticed that there is a significantly statistical similarity between the bursts from SGR 1806-20 and microglitches observed from the Vela pulsar with $\left|\frac{\delta v}{v}\right| \sim 10^{-9}$. Thus we see that these early statistical studies of bursts agree with our theory for bursts in magnetars. Even more, we shall show that after a giant glitch there is intense burst activity very similar to the settling earthquakes following a strong earthquake.

\subsection{Braking glitches}

In Sect. 4 we found that magnetic glitches in p-stars lead to:

$\frac{\delta \dot{v}}{\dot{v}} \simeq-\frac{\delta B_{\mathrm{c}}}{B_{\mathrm{c}}}>0$.

Since there is variation in both the inner core and the stellar radius, the moment of inertia of the star undergoes a variation of $\delta I$. It is easy to see that the increase in the inner core leads to an increase in the moment of inertia $I$; on the other hand, the reduction of the stellar radius implies $\delta I<0$. In a radio pulsar, where, by neglecting the variation of the magnetic energy, the conservation of the mass leads to Eq. (23), one can show that:

$\frac{\delta I}{I} \simeq \frac{\delta R}{R}<0$.

Moreover, from the conservation of angular momentum

$\frac{\delta I}{I}=-\frac{\delta v}{v}$

it follows that

$0<\frac{\delta v}{v} \simeq-\frac{\delta R}{R} \ll-\frac{\delta B_{\mathrm{c}}}{B_{\mathrm{c}}} \simeq \frac{\delta \dot{v}}{\dot{v}}$.

For magnetars, namely p-stars with a super strong magnetic field, the variation in magnetic energy can no be longer neglected. In this case, since the magnetic energy decreases, we find that the surface contraction in magnetars is smaller than in radio pulsars, which means that Eq. (24) even holds for magnetars. Moreover, since in radio pulsars we know that

$\frac{\delta v}{v}=-\frac{\delta I}{I} \simeq-\frac{\delta R}{R} \lesssim 10^{-6}$,

we see that in magnetars the following bound must hold:

$-\frac{\delta R}{R} \lesssim 10^{-6}$.

As a consequence we may write:

$\frac{\delta I}{I}=\left(\frac{\delta I}{I}\right)_{\text {core }}+\left(\frac{\delta I}{I}\right)_{\text {surf }},\left(\frac{\delta I}{I}\right)_{\text {surf }} \simeq \frac{\delta R}{R}<0$.

As we show in a moment, the variation in the moment of inertia induced by the core is positive. Thus if the core contribution overcomes the surface contribution to $\delta I$, we have a braking glitch where $-\frac{\delta v}{v}=\frac{\delta P}{P}>0$.

We believe that the most compelling evidence to support our proposal comes from the anomalous X-ray pulsar AXP 1E 2259+586. As reported in Woods et al. (2004), the timing data show that a large glitch occurred in AXP 1E 2259+586 coinciding with the 2002 June giant burst. Remarkably, at the time of the giant flare on August 27, 1998 the soft gamma ray repeater SGR 1900+14 displayed 
a discontinuous spin-down that was consistent with a braking glitch (Woods et al. 1999b). Our theory is able to explain why AXP 1E 2259+586 displayed a normal glitch, while SGR 1900+14 suffered a braking glitch.

To see this, we recall the spin-down parameters of these pulsars:

\section{SGR $1900+14$}

$P \simeq 5.16 \mathrm{~s}, \dot{P} \simeq 1.1 \times 10^{-10}, B_{\mathrm{S}} \simeq 7.4 \times 10^{14}$ Gauss,

AXP 1E 2259+586

$P \simeq 6.98 \mathrm{~s}, \dot{P} \simeq 2.0 \times 10^{-14}, B_{\mathrm{S}} \simeq 1.2 \times 10^{13}$ Gauss.

For canonical magnetars with $M \simeq 1.4 M_{\odot}$ and radius $R \simeq$ $10 \mathrm{~km}$, we have $\sqrt{g H} \simeq 0.55 \mathrm{GeV}$. Then, using $1 \mathrm{GeV}^{2} \simeq$ $5.12 \times 10^{19}$ Gauss, we rewrite Eq. (1) as:

$B_{\mathrm{S}} \simeq 1.54 \times 10^{16}\left(\frac{R_{\mathrm{c}}}{R}\right)^{3}$ Gauss.

Combining Eqs. (42) and (43) we get:

$$
\begin{array}{ll}
\text { SGR } 1900+14 & \left(\frac{R_{\mathrm{c}}}{R}\right)^{3} \simeq 4.81 \times 10^{-2}, \\
\text { AXP 1E 2259+586 } & \left(\frac{R_{\mathrm{c}}}{R}\right)^{3} \simeq 0.78 \times 10^{-3} .
\end{array}
$$

According to Eqs. (35), (37), and (41), to evaluate the sudden variation of the frequency and frequency derivative, we need $\delta R$ and $\delta R_{\mathrm{c}}$. These parameters can be estimated from the energy released during the giant bursts. In the case of AXP 1E 2259+586, the giant June 2002 burst followed an intense burst activity that lasted for about one year. Assuming a distance of $3 \mathrm{kpc}$ to $1 \mathrm{E} 2259+586$, Woods et al. (2004) measured an energy release of $2.7 \times 10^{39} \mathrm{erg}$ and $2.1 \times 10^{41} \mathrm{erg}$ for the fast and slow decay intervals, respectively. Due to this uncertainty, we conservatively estimate the energy released during the giant burst to be:

$\mathrm{AXP} 1 \mathrm{E} 2259+586 \quad E_{\text {burst }} \simeq 1.0 \times 10^{40} \mathrm{erg}$.

On August 27, 1998 a giant burst from the soft gamma ray repeater SGR 1900+14 was recorded. The estimate energy released was:

SGR $1900+14 \quad E_{\text {burst }} \simeq 1.0 \times 10^{44} \mathrm{erg}$.

As already discussed in Sect. 4, the energy released during a burst in magnetars is given by the magnetic energy directly injected and dissipated into the magnetosphere, Eq. (34), which we rewrite as

$\delta E_{B}^{\mathrm{ext}} \simeq \frac{1}{3} R^{3} B_{\mathrm{S}}^{2} \frac{\delta B_{\mathrm{S}}}{B_{\mathrm{S}}} \simeq 2.6 \times 10^{44} \mathrm{erg}\left(\frac{B_{\mathrm{S}}}{10^{14} \text { Gauss }}\right)^{2} \frac{\delta B_{\mathrm{S}}}{B_{\mathrm{S}}}$,

so that by combining Eqs. (47), (46), (45), and (42) we get:

$$
\begin{array}{ll}
\text { SGR 1900+14 } & \frac{\delta B_{\mathrm{S}}}{B_{\mathrm{S}}} \simeq \frac{\delta R_{\mathrm{c}}}{R_{\mathrm{c}}} \simeq 0.70 \times 10^{-2} \\
\text { AXP 1E 2259+586 } & \frac{\delta B_{\mathrm{S}}}{B_{\mathrm{S}}} \simeq \frac{\delta R_{\mathrm{c}}}{R_{\mathrm{c}}} \simeq 0.27 \times 10^{-2}
\end{array}
$$

Thus, according to Eq. (35) we may estimate the sudden variation of $\dot{v}$ as

$\frac{\delta \dot{v}}{\dot{v}} \simeq 2 \frac{\delta R_{\mathrm{c}}}{R_{\mathrm{c}}} \sim 10^{-2}$,

for both glitches. On the other hand, we have:

$\left(\frac{\delta I}{I}\right)_{\text {core }} \simeq \frac{15}{2} \frac{\varepsilon_{\mathrm{c}}-\varepsilon}{\bar{\varepsilon}}\left(\frac{R_{\mathrm{c}}}{R}\right)^{5} \frac{\delta R_{\mathrm{c}}}{R_{\mathrm{c}}} \simeq \frac{15}{2}\left(\frac{R_{\mathrm{c}}}{R}\right)^{5} \frac{\delta R_{\mathrm{c}}}{R_{\mathrm{c}}}$,

leading to:

SGR $1900+14$

$$
\left(\frac{\delta I}{I}\right)_{\text {core }} \simeq 3.34 \times 10^{-4}
$$

$\mathrm{AXP} 1 \mathrm{E} 2259+586 \quad\left(\frac{\delta I}{I}\right)_{\text {core }} \simeq 1.34 \times 10^{-7}$.

On the other hand, we expect that during the giant glitch $\left(\frac{\delta I}{I}\right)_{\text {surf }} \sim 10^{-6}$. As a consequence, for AXP 1E 2259+586 the core contribution is negligible with respect to the surface contribution to $\delta I$. In other words, AXP 1E 2259+586 displays a normal glitch with $\frac{\delta v}{v} \sim 10^{-6}$. On the contrary, Eq. (51) indicates that SGR $1900+14$ suffered a braking glitch with $\frac{\delta v}{v} \sim-3.34 \times 10^{-4}$, giving:

$\Delta P \simeq 1.72 \times 10^{-3} \mathrm{~s}$.

We see that our theory agrees with observations, for a glitch of size $\frac{\delta v}{v}=4.24(11) \times 10^{-6}$ was observed in AXP 1E 2259+586, and it preceded the burst activity (Woods et al. 2004). Moreover, our theory predicts a sudden increase of the spindown torque according to Eq. (49). In Woods et al. (2004) it is pointed out that it was not possible to give a reliable estimate of the variation of the frequency derivative since the pulse profile was undergoing large changes, thus compromising the phase alignment with the pulse profile template. As discussed in Sect. 5.1, soon after the giant burst AXP 1E 2259+586 indeed suffered an intense burst activity. Now, according to our theory, during the burst activity there is both a continuous injection of magnetic energy into the magnetosphere and variation of the magnetic torque explaining the anomalous timing noise observed in 1E 2259+586. In addition, Woods et al. (1999b) reported a gradual increase in the nominal spin-down rate and a discontinuous spin down event associated with the 1998 August 27 flare from SGR 1900+14. Extrapolating the long-term trends found before and after August 27, they found evidence of a braking glitch with $\Delta P \simeq 0.57(2) \times 10^{-3} \mathrm{~s}$. In view of our theoretical uncertainties, the agreement with our Eq. (52) is rather good.

To our knowledge the only attempt to explain within the standard model the braking glitch as observed in SGR 1900+14 is done in Thompson et al. (2000) where it is suggested that the violent August 27 event involved a glitch. The magnitude of the glitch was estimated by scaling to the largest glitches in young, active pulsars with similar spin-down ages and internal temperature. In this way they deduced the estimate $\left|\frac{\Delta P}{P}\right| \sim 10^{-5}$ to $10^{-4}$. However, this explanation overlooks the well-known fact that radio pulsars display normal glitches and no braking glitches. Moreover, there are no arguments to explain why AXP 1E 2259+586 displayed a normal glitch instead of a braking glitch. 
Let us conclude this section by briefly discussing the December 27, 2004 giant flare from SGR 1806-20. During this tremendous outburst SGR 1806-20 released a huge amount of energy, $E_{\text {burst }} \sim 10^{46} \mathrm{erg}$. Using the spin-down parameters reported in Mereghetti et al. (2005a):

SGR $1806-20$

$P \simeq 7.55 \mathrm{~s}, \dot{P} \simeq 5.5 \times 10^{-10}, B_{\mathrm{S}} \simeq 2.0 \times 10^{15}$ Gauss,

we find:

SGR $1806-20 \quad \frac{\delta B_{\mathrm{S}}}{B_{\mathrm{S}}} \simeq \frac{\delta R_{\mathrm{c}}}{R_{\mathrm{c}}} \simeq 9.6 \times 10^{-2}$.

Thus, we predict that SGR 1806-20 should display a gigantic braking glitch with $\frac{\Delta P}{P} \simeq 2.4 \times 10^{-2}$, or:

$\Delta P \simeq 1.8 \times 10^{-1} \mathrm{~s}$.

\subsection{Quiescent luminosity}

The basic mechanism to explain the quiescent X-ray emission in our magnetars is the internal dissipation of magnetic energy. Our mechanism is basically the same as in the standard magnetar model based on a neutron star (Duncan \& Thompson 1996). Below, we compare our proposal with the standard theory.

In Sect. 4 we showed that during a glitch there is a huge amount of magnetic energy released into the magnetar:

$-\delta E_{B}^{\mathrm{int}} \simeq \frac{1}{3} R^{3} B_{\mathrm{S}}^{2}\left(\frac{R}{R_{\mathrm{c}}}\right)^{3} \frac{\delta R_{\mathrm{c}}}{R_{\mathrm{c}}}$.

As in the previous section, we use SGR $1900+14$ and AXP 1E $2259+586$ as prototypes for soft gamma ray repeaters and anomalous X-ray pulsars, respectively. Using the results of Sect. 4.1, we find:

$$
\begin{array}{ll}
\text { SGR 1900+14 } & -\delta E_{B}^{\mathrm{int}} \simeq 3.0 \times 10^{47} \operatorname{erg} \frac{\delta R_{\mathrm{c}}}{R_{\mathrm{c}}}, \\
\text { AXP 1E 2259+586 } & -\delta E_{B}^{\mathrm{int}} \simeq 4.8 \times 10^{45} \mathrm{erg} \frac{\delta R_{\mathrm{c}}}{R_{\mathrm{c}}} .
\end{array}
$$

This release of magnetic energy is dissipated, leading to observable surface luminosity. To see this, we need a thermal evolution model that calculates the interior temperature distribution. In the case of neutron stars, such a calculation has been performed in Van Riper (1991), where it is shown that the isothermal approximation is a rather good approximation in the range of inner temperatures of interest. The equation that determines the thermal history of a p-star was discussed in Cea (2004a) in the isothermal approximation

$C_{V} \frac{\mathrm{d} T}{\mathrm{~d} t}=-\left(L_{\nu}+L_{\gamma}\right)$,

where $L_{\nu}$ is the neutrino luminosity, $L_{\gamma}$ the photon luminosity, and $C_{V}$ the specific heat. Assuming blackbody photon emission from the surface at an effective surface temperature $T_{\mathrm{S}}$ we have

$L_{\gamma}=4 \pi R^{2} \sigma_{\mathrm{SB}} T_{\mathrm{S}}^{4}$, where $\sigma_{\mathrm{SB}}$ is the Stefan-Boltzmann constant. In Cea (2004a) we assumed that the surface and interior temperature were related by:

$\frac{T_{\mathrm{S}}}{T}=10^{-2} a$.

Equation (60) is relevant for a p-star that is not bare, namely for $\mathrm{p}$-stars that are endowed with a thin crust. The vacuum gap between the core and the crust, which is of the order of several hundred fermis, leads to a strong suppression of the surface temperature with respect to the core temperature. The precise relation between $T_{\mathrm{S}}$ and $T$ could be obtained by a careful study of the crust and core thermal interaction. In any case, our phenomenological relation Eq. (60) allows a wide variation in $T_{\mathrm{S}}$, which encompasses the neutron star relation (see, for instance, Gundmundsson et al. 1983). Moreover, our cooling curves display a rather weak dependence on the parameter $a$ in Eq. (60). Since we are interested in the quiescent luminosity, we do not need to know the precise value of this parameter, so in the following we shall assume $a \sim 1$. In other words, we assume:

$T_{\mathrm{S}} \simeq 10^{-2} T$.

Obviously, parameter $a$ is relevant to evaluating the surface temperature once the core temperature is given. In the relevant range of core temperature $T \sim 10^{8} \mathrm{~K}$, our Eq. (61) is practically identical to the parametrization adopted in Duncan \& Thompson (1996) within the standard magnetar model:

$T_{\mathrm{S}} \simeq 1.3 \times 10^{6} \mathrm{~K}\left(\frac{T}{10^{8} \mathrm{~K}}\right)^{\frac{5}{9}}$.

The neutrino luminosity $L_{v}$ in Eq. (58) is given by the direct $\beta$-decay quark reactions, the dominant cooling processes by neutrino emission. From the results in Cea (2004a), we find:

$L_{\nu} \simeq 1.22 \times 10^{36} \frac{\mathrm{erg}}{\mathrm{s}} T_{9}^{8}$,

where $T_{9}$ is the temperature in units of $10^{9} \mathrm{~K}$. Note that the neutrino luminosity $L_{v}$ has the same temperature dependence as the neutrino luminosity by modified URCA reactions in neutron stars, but it is more than two orders of magnitude smaller. From the cooling curves reported in Cea (2004a) we infer that the surface and interior temperature are almost constant up to time $\tau \sim 10^{5}$ years. Observing that magnetars candidates are rather young pulsars with $\tau_{\text {age }} \lesssim 10^{5}$ years, we may estimate the average surface luminosities as:

$L_{\gamma} \simeq \frac{-\delta E_{B}^{\mathrm{int}}}{\tau_{\mathrm{age}}}$

We assume $\tau_{\text {age }} \approx \tau_{\mathrm{c}}$ for SGR $1900+14$. On the other hand, it is known that for AXP 1 E $2259+586 \tau_{\text {age }} \sim 10^{3}$ years $\ll \tau_{c}$, so we get:

$\begin{array}{ll}\text { SGR } 1900+14 & L_{\gamma} \simeq 1.3 \times 10^{37} \frac{\mathrm{erg}}{\mathrm{s}} \frac{\delta R_{\mathrm{c}}}{R_{\mathrm{c}}}, \\ \text { AXP 1E 2259+586 } & L_{\gamma} \simeq 1.5 \times 10^{35} \frac{\mathrm{erg}}{\mathrm{s}} \frac{\delta R_{\mathrm{c}}}{R_{\mathrm{c}}} .\end{array}$

We see that it is enough to assume that in the past SGR $1900+14$ suffered a glitch with $\frac{\delta R_{\mathrm{c}}}{R_{\mathrm{c}}} \sim 10^{-2}$ to sustain the 
observed luminosity $L_{\gamma} \sim 10^{35} \frac{\mathrm{erg}}{\mathrm{s}}$ (assuming a distance of about $10 \mathrm{kpc}$ ). In the case of AXP 1E 2259+586, assuming a distance of about $3 \mathrm{kpc}$, the observed luminosity is $L_{\gamma} \sim$ $10^{34} \frac{\mathrm{erg}}{\mathrm{s}}$, so that we infer that in the past this pulsar had suffered a giant glitch with $\frac{\delta R_{\mathrm{c}}}{R_{\mathrm{c}}} \sim 10^{-1}$, quite similar to the recent SGR 1806-20 glitch.

Within the range of validity of our approximation, Eq. (64) is valid as long as $L_{\gamma}$ dominates $L_{\gamma}$, otherwise the star is efficiently cooled by neutrino emission and the surface luminosity saturates to $L_{\gamma}^{\max }$. We may quite easily evaluate this limiting luminosity from $L_{\gamma}^{\max } \simeq L_{v}$. Using Eq. (61) and $R \simeq 10 \mathrm{~km}$, we get:

$L_{\gamma}^{\max } \simeq 4.2 \times 10^{37} \frac{\mathrm{erg}}{\mathrm{s}}$.

Since our neutrino luminosity is reduced by more than two orders of magnitude with respect to neutron stars, $L_{\gamma}^{\max }$ is about two orders of magnitude greater than the maximum allowed surface luminosity in neutron stars (Van Riper 1991). Thus, our theory allows us to easily account for observed luminosities up to $10^{36} \frac{\mathrm{erg}}{\mathrm{s}}$.

As already discussed, the origin of the quiescent emission is the huge release of magnetic energy in the interior of the magnetar. Our previous estimate of the quiescent luminosities assumed that the interior temperature distribution was uniform. However, due to the huge magnetic field, the thermal conductivity is enhanced along the magnetic field. This turns out to be the case for both electron and quarks, since we argued that the magnetic and chromomagnetic fields are aligned. As a consequence, we expect that the quiescent spectrum should be parameterized as two blackbodies with parameter $R_{1}, T_{1}$ and $R_{2}, T_{2}$, respectively. Since the blackbody luminosities $L_{\gamma 1}$ and $L_{\gamma 2}$ fit in the same order of magnitude, our previous estimates for the quiescent luminosities are unaffected. Moreover, since the thermal conductivity is enhanced along the magnetic field, the high temperature blackbody, with temperature $T_{2}$, originates from the heated polar magnetic cups. Thus we have:

$R_{1} \lesssim R, \quad R_{2} \lesssim 1 \mathrm{~km}$,

$T_{2}>T_{1}, \quad R_{1}>R_{2}, \quad L_{\gamma 1} \simeq L_{\gamma 2}$.

Note that there is a natural anticorrelation between blackbody radii and temperatures.

It is customary to fit the quiescent spectrum of anomalous X-ray pulsars and soft gamma ray repeaters in terms of a blackbody plus power law. In particular, it is assumed that the power law component extends to energy greater than an arbitrary cutoff energy $E_{\text {cutoff }} \simeq 2 \mathrm{keV}$. It is worthwhile noting that these parameterizations of the quiescent spectra are in essence phenomenological fits. Indeed, within the standard magnetar model (Duncan \& Thompson 1996), the power law should be related to a hydromagnetic wind accelerated by Alfven waves. The luminosity of the wind emission should increase with magnetic field strength as $L_{\text {wind }} \simeq L_{\mathrm{PL}} \sim B_{\mathrm{S}}^{2}$. On the other hand, the blackbody luminosities should scale as $B_{\mathrm{S}}^{4.4}$ (Duncan \& Thompson 1996). Thus, the ratio $L_{\mathrm{PL}} / L_{\mathrm{BB}}$ decreases with increasing magnetic field strengths, contrary to observations (Marsden \& White 2001). In our theory well-defined physical arguments lead to the two blackbody representations of the quiescent spectra, whose parameters are constrained by our Eq. (67). As a matter of fact, we have checked in the literature that the quiescent spectra of both anomalous X-ray pulsars and soft gamma ray repeaters could be accounted for by two balckbodies. For instance, in Morii et al. (2003) the quiescent spectrum of AXP 1E 1841-045 is fitted well with the standard power law plus blackbody (reduced $\chi^{2} /$ d.o.f. $\simeq 1.11$ ), nevertheless the two blackbody model also gives a rather good fit (reduced $\chi^{2} /$ d.o.f. $\simeq 1.12$ ). Interestingly enough the blackbody parameters

$R_{1}=5.7{ }_{-0.5}^{+0.6} \mathrm{~km}, \quad T_{1}=0.47 \pm 0.02 \mathrm{keV}$,

$R_{2}=0.36_{-0.07}^{+0.08} \mathrm{~km}, \quad T_{2}=1.5_{-0.1}^{+0.2} \mathrm{keV}$,

are in agreement with Eq. (67). Moreover, assuming that the power law component in the standard parametrization of quiescent spectra account for the hot blackbody component in our parametrization, we find that the suggestion $L_{\gamma 1} \simeq L_{\gamma 2}$ in Eq. (67) agrees with observations (Marsden \& White 2001).

Another interesting consequence of the anisotropic distribution of the surface temperature due to strong magnetic fields is that the thermal surface blackbody radiation will be modulated by the stellar rotation. As a matter of fact, Ozel (2002) argues that the observed properties of anomalous X-ray pulsars can be accounted for by magnetars with a single hot region. It is remarkable that our interpretation naturally explains the observed change in pulse profile of SGR $1900+14$ following the August 27, 1998 giant flare. It seem that our picture is in fair qualitative agreement with several observations. However, any further discussion of this matter goes beyond the aim of the present paper.

\subsection{Bursts}

In the present section we discuss how glitches in our magnetars give rise to the burst activity from soft gamma-ray repeaters and anomalous X-ray pulsars. We said in Sect. 4 that the energy released during a burst in a magnetar is given by the magnetic energy directly injected into the magnetosphere, Eq. (34).

Before addressing the problem of the dissipation of this magnetic energy in the magnetosphere, let us discuss the observational signatures at the onset of the burst. Observations indicate that at the onset of giant bursts the flux displays a spike with a very short rise time $t_{1}$ followed by a rapid but more gradual decay time $t_{2}$. According to our previous discussion, the onset of bursts is due to the positive variation in the surface magnetic field $\delta B_{\mathrm{S}}$, which in turn implies a sudden increase of the magnetic energy stored in the magnetosphere. Now, according to Eq. (33) we see that almost all the magnetic energy is stored in the region

$R \lesssim r \lesssim 10 R$

so that the rise time is essentially the time needed to propagate the information in the magnetosphere that the surface magnetic field is varied. Then we are lead to

$t_{1} \simeq 9 R \simeq 3 \times 10^{-4} \mathrm{~s}$ 
which indeed agrees with observations. On the other hand, in our proposal the decay time $t_{2}$ depends on the physical properties of the magnetosphere. It is natural to identify $t_{2}$ with the time needed for the system to react to the sudden variation of the magnetic field. In other words, we may consider the magnetosphere as a huge electric circuit that is subject to a sudden increase in power from some external device. The electric circuit reacts to the external injection of energy within a transient time. Thus, in our case the time $t_{2}$ is a function of the geometry and the conducting properties of the magnetosphere. In general, it is natural to expect that $t_{1} \ll t_{2}$, and the time extension of the initial spike is:

$\delta t_{\text {spike }} \simeq t_{2}-t_{1} \simeq t_{2}$.

Remarkably, observations show that the observed giant bursts are characterized by almost the same $\delta t_{\text {spike }}$,

$\delta t_{\text {spike }} \simeq t_{2} \simeq 0.1 \mathrm{~s}$,

signalling that the structures of the magnetosphere of soft gamma-ray repeaters and anomalous X-ray pulsars are very similar.

Since the magnetic field is varied by $\delta B_{\mathrm{S}}$ in a time $\delta t_{\text {spike }}$, then from Maxwell equations it follows that it must be an induced electric field. To see this, let us consider the dipolar magnetic field in the polar coordinate:

$B_{r}=-\frac{2 B_{\mathrm{S}} R^{3} \cos \theta}{r^{3}}$

$B_{\theta}=-\frac{B_{\mathrm{S}} R^{3} \sin \theta}{r^{3}}$

$B_{\varphi}=0$.

Thus, observing that $\frac{\delta B_{\mathrm{S}}}{\delta t_{\text {spike }}}$ is the time derivative of the magnetic field it is easy to find the induced azimuthal electric field:

$E_{\varphi}=+\frac{\delta B_{\mathrm{S}}}{\delta t_{\text {spike }}} \frac{R^{3} \sin \theta}{r^{2}}, \quad r \geq R$

To discuss the physical effects of the induced azimuthal electric field Eq. (74), it is convenient to work in the co-rotating frame of the star. We assume that the magnetosphere contains a neutral plasma. Thus, we see that charges are suddenly accelerated by the huge induced azimuthal electric field $E_{\varphi}$ and thereby acquire an azimuthal velocity $v_{\varphi} \simeq 1$ that is directed along the electric field for positive charges and in the opposite direction for negative charges. It is well known that relativistic, charged particles moving in the magnetic field $\boldsymbol{B}(\boldsymbol{r})$, Eq. (73), will emit synchrotron radiation (see for instance Wallace 1977; Ginzburg 1979). Below we show that these processes are able to completely dissipate the whole magnetic energy injected into the magnetosphere following a glitch.

The charges accelerated by the electric field $E_{\varphi}$ are subject to the drift Lorentz force $\boldsymbol{F}=q \boldsymbol{v}_{\varphi} \times \boldsymbol{B}(\boldsymbol{r})$, whose radial component is

$F_{r}=-q v_{\varphi} B_{\theta} \simeq+q v_{\varphi} B_{\mathrm{S}} \sin \theta\left(\frac{R}{r}\right)^{3}$,

while the $\theta$ component is:

$F_{\theta}=+q v_{\varphi} B_{r} \simeq-2 q v_{\varphi} B_{\mathrm{S}} \cos \theta\left(\frac{R}{r}\right)^{3}$.
The radial component $F_{r}$ pushes both positive and negative charges radially outward. Then, we see that the plasma in the outermost part of the magnetosphere is subject to an intense radial centrifugal force, so that the plasma must flow radially outward giving rise to a blast wave. On the other hand, $F_{\theta}$ is centripetal in the upper hemisphere and centrifugal in the lower hemisphere. As a consequence, in the lower hemisphere, charges are pushed towards the magnetic equatorial plane $\cos \theta=0$, while the centripetal force gives rise to a rather narrow jet along the magnetic axis in the upper hemisphere (the north magnetic pole). As a consequence, at the onset of the giant burst there is an almost spherically symmetric outflow from the pulsar, together with a collimated jet from the north magnetic pole.

Interestingly enough, a fading radio source has been seen from SGR 1900+14 following the August 271998 giant flare (Frail et al. 1999). The radio afterglow is consistent with an outflow expanding subrelativistically into the surrounding medium. This agrees with our model once one takes into account that the azimuthal electric field is rapidly decreasing with the distance from the star, so that $v_{\varphi} \lesssim 1$ for the plasma in the outer region of the magnetosphere. However, we believe that the most compelling evidence in favor of our proposal comes from the detected radio afterglow following the 27 December 2004 gigantic flare from SGR 1806-20 (Gaensler et al. 2005; Cameron et al. 2005; Wang et al. 2005; Granot et al. 2006; Gelfand et al. 2005). In fact, the fading radio source from SGR 1806-20 has similar properties to those observed from SGR 1900+14, but much higher energy. Interesting in this case is that the spectra of the radio afterglow clearly showed the presence of the expected spherical, non relativistic expansion, together with a sideways expansion of a jetted explosion (see Fig. 1 in Gaensler et al. 2005; and Fig. 1 in Cameron et al. 2005). Finally, the lower limit of the outflow $E \gtrsim 10^{44.5} \mathrm{erg}$ (Gelfand et al. 2005) implies that the blast wave and the jet dissipate only a small fraction of the burst energy, which is about $10^{46}$ erg (see Sect. 4.1). Thus, we infer that almost all the burst energy must be dissipated into the magnetosphere.

In the co-rotating frame of the star the plasma, at rest before the onset of the burst, is suddenly accelerated by the induced electric field, thereby acquiring an azimuthal velocity $v_{\varphi} \simeq 1$. Now, relativistic charges are moving in the dipolar magnetic field of the pulsar, so they will lose energy by emitting synchrotron radiation until they come to rest. Of course, this process involving charges that are distributed in the whole magnetosphere will last for a time $t_{\text {dis }}$ that is much longer than $\delta t_{\text {spike }}$. Actually, $t_{\text {dis }}$ will depend on the injected energy, the plasma distribution, and the magnetic field strength. Moreover, one should also watch out for repeated charge and photon scatterings, so that it is not easy to estimate $t_{\mathrm{dis}}$ without a precise knowledge of the pulsar magnetosphere. At the same time, the fading of the luminosity with time, the so-called light curve $L(t)$, cannot be determined without a precise knowledge of the microscopic dissipation mechanisms. However, since the dissipation of the magnetic energy involves the whole magnetosphere, it turns out that we may accurately reproduce the time variation of $L(t)$ without a precise knowledge of the microscopic dissipative mechanisms. In Sect. 5 we develop an effective description 
where our ignorance of the microscopic dissipative processes is encoded in a few macroscopic parameters, which then allows us to determine the light curves.

In order to investigate the spectral properties of the luminosity, we need to consider the synchrotron radiation spectral distribution. Since radiation from electrons is far more important than from protons, we focus on electrons. It is well known that the synchrotron radiation will be mainly at the frequencies (Wallace 1977; Ginzburg 1979)

$\omega_{m} \simeq \gamma^{2} \frac{e B}{m_{\mathrm{e}}}$,

where $\gamma$ is the electron Lorentz factor. Using Eq. (73) we get:

$\omega(r) \simeq \gamma^{2} \frac{e B_{\mathrm{S}}}{m_{\mathrm{e}}}\left(\frac{R}{r}\right)^{3}, R \lesssim r \lesssim 10 R$.

As it is useful to numerically estimate the involved frequencies, we consider the giant flare of August 27, 1998 from SGR 1900+14:

$B_{\mathrm{S}} \simeq 7.4 \times 10^{14}$ Gauss, $\quad \frac{\delta B_{\mathrm{S}}}{B_{\mathrm{S}}} \simeq 10^{-2}$.

From Eq. (78) it follows that

$\omega(r) \simeq \gamma^{2} 8.67 \mathrm{MeV}\left(\frac{R}{r}\right)^{3}, R \lesssim r \lesssim 10 R$,

or

$\omega_{1} \simeq \gamma^{2} 8.67 \mathrm{keV} \lesssim \omega \lesssim \omega_{2} \simeq \gamma^{2} 8.67 \mathrm{MeV}$.

The power injected into the magnetosphere is supplied by the azimuthal electric field during the initial hard spike. To estimate the total power, we therefore need to evaluate the power supplied by the azimuthal electric field. By considering the infinitesimal volume $\mathrm{d} V=r^{2} \sin \theta \mathrm{d} r \mathrm{~d} \theta \mathrm{d} \varphi$, the power supplied by the induced electric field $E_{\varphi}$ in $\mathrm{d} V$ is

$\mathrm{d} \dot{W}_{E_{\varphi}} \simeq n_{\mathrm{e}} e \frac{\delta B_{\mathrm{S}}}{\delta t_{\text {spike }}} v_{\varphi} R^{3} \sin ^{2} \theta \mathrm{d} r \mathrm{~d} \theta \mathrm{d} \varphi$

where $n_{\mathrm{e}}$ is the electron number density. Since the magnetosphere is axially symmetric, it follows that $n_{\mathrm{e}}$ cannot depend on $\varphi$. Moreover, within our theoretical uncertainties we may neglect the dependence on $\theta$, so that, integrating over $\theta$ and $\varphi$ we get:

$\mathrm{d} \dot{W}_{E_{\varphi}} \simeq 2 \pi^{2} n_{\mathrm{e}} e \frac{\delta B_{\mathrm{S}}}{\delta t_{\text {spike }}} v_{\varphi} R^{3} \mathrm{~d} r$.

In order to determine the spectral distribution of the supplied power, we note that to a good approximation all the synchrotron radiation is emitted at $\omega_{m}$, Eq. (77), so we may use Eq. (80) to get:

$-\mathrm{d} r \simeq \frac{R}{3} \gamma^{\frac{2}{3}}\left(\frac{e B_{\mathrm{S}}}{m_{\mathrm{e}}}\right)^{1 / 3} \frac{1}{\omega^{\frac{4}{3}}} \mathrm{~d} \omega$.

Inserting Eqs. (84) into (83) we obtain the spectral power

$F(\omega) \mathrm{d} \omega \simeq \frac{2 \pi^{2}}{3} n_{\mathrm{e}} e \frac{\delta B_{\mathrm{S}}}{\delta t_{\text {spike }}} v_{\varphi} R^{4} \gamma^{\frac{2}{3}}\left(\frac{e B_{\mathrm{S}}}{m_{\mathrm{e}}}\right)^{1 / 3} \frac{1}{\omega^{\frac{4}{3}}} \mathrm{~d} \omega$, while the total luminosity is given by:

$L=\int_{\omega_{1}}^{\omega_{2}} F(\omega) \mathrm{d} \omega$.

Note that $L$ is the total luminosity injected into the magnetosphere during the initial hard spike. Since the spike lasts for $\delta t_{\text {spike }}$, we have

$E_{\text {burst }} \simeq \delta t_{\text {spike }} L$,

where $E_{\text {burst }}$ is the total burst energy. In the case of the August 27, 1998 giant burst from SGR 1900+14, the burst energy is given by Eq. (46). Thus, using Eqs. (87) and (72) we have:

$L \simeq 10^{45} \frac{\mathrm{erg}}{\mathrm{s}}$,

which, indeed, agrees with observations.

It is worthwhile estimating the electron number density needed to dissipate the magnetic energy injected in the magnetosphere. To this end, we assume a uniform number density. Thus, using Eqs. (86), (85), and (81) we get

$L \simeq 18 \pi^{2} n_{\mathrm{e}} e \frac{\delta B_{\mathrm{S}}}{\delta t_{\text {spike }}} R^{4}$

where we used $v_{\varphi} \simeq 1$. Specializing to the August 27 giant burst we find:

$n_{\mathrm{e}} \simeq 2.0 \times 10^{14} \mathrm{~cm}^{-3}$,

indeed a reasonable value. Soon after the initial spike, the induced azimuthal electric field vanishes and the luminosity decreases due to dissipative processes in the magnetosphere. As thoroughly discussed in Sect. 5, it is remarkable that the fading of the luminosity can be accurately reproduced without a precise knowledge of the microscopic dissipative mechanisms. Thus, by combining the time evolution of the luminosity $L(t)$ with the spectral decomposition we may obtain the time evolution of the spectral components. Starting from Eq. (85) the spectral luminosities can be accounted for by two blackbodies and a power law, and then we discuss the time evolution of the three different spectral components.

The spectral decomposition Eq. (85) seems to indicate that the synchrotron radiation follows a power law distribution. However, one should watch for reprocessing effects that redistribute the spectral distribution. We note that photons with energy $\omega \geq 2 m_{\mathrm{e}}$ quickly will copiously produce almost relativistic $\mathrm{e}^{ \pm}$pairs. Following Duncan \& Thompson (1995), it is easy to argue that the energy of relativistic particles is rapidly converted due to comptonization to thermal photon-pair plasma, even if the particles are injected steadily in a time $\delta t_{\text {spike. Since }}$ the pair production is quite close to the stellar surface, we may adopt the rather crude approximation of a uniform magnetic field $B \simeq B_{\mathrm{S}}$ throughout the volume $V_{\text {plasma }} \simeq 12 \pi R^{3}$ (Duncan $\&$ Thompson 1995). Since typical magnetic fields in magnetars are well above $B_{\mathrm{QED}}$, electrons and positrons sit in the lowest Landau levels. In this approximation we deal with an almost 
one-dimensional pair plasma whose energy density is (Duncan \& Thompson 1995):

$$
\begin{aligned}
u_{\mathrm{e}} & \simeq m_{\mathrm{e}}\left(n_{\mathrm{e}^{+}}+n_{\mathrm{e}^{-}}\right) \\
& \simeq \frac{2}{(2 \pi)^{\frac{3}{2}}} e B_{\mathrm{S}} m_{\mathrm{e}}^{2}\left(\frac{T_{\text {plasma }}}{m_{\mathrm{e}}}\right)^{\frac{1}{2}} \exp \left(-\frac{m_{\mathrm{e}}}{T_{\text {plasma }}}\right),
\end{aligned}
$$

for $T_{\text {plasma }}<m_{\mathrm{e}}$, where $T_{\text {plasma }}$ is the plasma temperature. Thus, the total energy density of the thermal photon-pair plasma is:

$u=u_{\mathrm{e}}+u_{\gamma} \simeq u_{\mathrm{e}}+\frac{\pi^{2}}{15} T_{\text {plasma }}^{4}$.

The plasma temperature is determined by equating the thermal energy Eq. (92) with the fraction of burst energy released in the spectral region $\omega \geq 2 m_{\mathrm{e}}$. It is easy to find:

$E_{\text {pairs }} \simeq 0.147 E_{\text {burst }}$,

where for the numerical estimate we approximated $\omega_{1} \simeq$ $10 \mathrm{keV}$ and $\omega_{2} \simeq 10 \mathrm{MeV}$, corresponding to mildly relativistic electrons in the magnetosphere. From that we have:

$$
\begin{array}{r}
\frac{2}{(2 \pi)^{\frac{3}{2}}} e B_{\mathrm{S}} m_{\mathrm{e}}^{2}\left(\frac{T_{\text {plasma }}}{m_{\mathrm{e}}}\right)^{\frac{1}{2}} \exp \left(-\frac{m_{\mathrm{e}}}{T_{\text {plasma }}}\right) \\
+\frac{\pi^{2}}{15} T_{\text {plasma }}^{4} \simeq \frac{E_{\text {pairs }}}{V_{\text {plasma }}} .
\end{array}
$$

In the case of the August 27 giant burst from SGR 1900+14 we find

$$
\sqrt{x} \exp \left(-\frac{1}{x}\right)+0.311 x^{4} \simeq 1.32 \times 10^{-2}, \quad x=\frac{T_{\text {plasma }}}{m_{\mathrm{e}}},
$$

whose solution gives $T_{\text {plasma }} \simeq 135 \mathrm{keV}$.

However, this is not the end of the story. Indeed, our thermal photon-pair plasma at temperature $T_{\text {plasma }}$ will be reprocessed by thermal electrons on the surface, which are at the temperature of the thermal quiescent emission $T_{\mathrm{Q}} \lesssim 1 \mathrm{keV}$. Thus, photons at temperature $T_{\text {plasma }} \gg T_{\mathrm{Q}}$ are rapidly cooled by Thompson scattering off electrons in the stellar atmosphere, that extends over several hundreds fermis beyond the edge of the star. The rate of change of the radiation energy density is given by (for instance, see Peebles 1993):

$\frac{1}{u_{\gamma}} \frac{\mathrm{d} u_{\gamma}}{\mathrm{d} t} \simeq \frac{4 \sigma_{T} n_{\mathrm{Q}}}{m_{\mathrm{e}}}\left(T_{\mathrm{Q}}-T_{\text {plasma }}\right)$,

where $n_{\mathrm{Q}}$ is the number density of electrons in the stellar atmosphere. The electron number density in the atmosphere of a p-star is on the same order as in strange stars, where $n_{\mathrm{Q}} \simeq 10^{33} \mathrm{~cm}^{-3}$ (Alcock et al. 1986). Due to the very high electron density of electrons near the surface of the star, the thermal photon-pair plasma is efficiently cooled to a final temperature that is much less than $T_{\text {plasma }}$. At the same time, the energy transferred to the stellar surface leads to an increase in the effective quiescent temperature. Therefore we are lead to conclude that the quiescent luminosity must increase during the burst activity.

Let $T_{1}$ be the final plasma temperature, then we see that the thermal photon-pair plasma contribution to the luminosity can be accounted for with an effective blackbody with temperature $T_{1}$ and radius $R_{1}$ on the order of the stellar radius. As a consequence the resulting blackbody luminosity is:

$L_{1}=4 \pi R_{1}^{2} \sigma_{\mathrm{SB}} T_{1}^{4}, \quad R_{1} \lesssim R$.

In general, the estimate of the effective blackbody temperature $T_{1}$ is quite difficult. However, according to Eq. (93) we known that $L_{1}$ must account for about 0.147 of the total luminosity. So that we have:

$L_{1}(t) \simeq 0.147 L(t)$.

This last equation allows us to determine the blackbody temperature. For instance, soon after the hard spike we have $L(0) \simeq$ $\frac{E_{\text {burst }}}{\delta t_{\text {spike }}} \simeq 10^{45} \frac{\mathrm{erg}}{\mathrm{s}}$ for the giant burst from SGR $1900+14$. Thus, using $R_{1} \simeq R$, from Eq. (98) we get:

$T_{1}(0) \simeq 61 \mathrm{keV}$,

with surface luminosity $L_{1}(0) \simeq 10^{44} \frac{\mathrm{erg}}{\mathrm{s}}$.

Let us consider the remaining spectral power with $\omega \lesssim 2 m_{\mathrm{e}}$. We recall that the spectral power Eq. (85) originates from the power supplied by the induced electric field Eq. (83). It is evident from Eq. (83) that, as long as $v_{\varphi} \simeq 1$, the power supplied by the electric field $E_{\varphi}$ does not depend on the mass of accelerated charges. Since the plasma in the magnetosphere is neutral, it follows that protons acquire the same energy as electrons. On the other hand, since the protons' synchrotron frequencies are reduced by a factor $\frac{m_{\mathrm{e}}}{m_{\mathrm{p}}}$, the protons will emit synchrotron radiation near $\omega_{1}$. As a consequence, photons with frequencies near $\omega_{1}$ suffer resonant synchrotron scattering, which considerably redistributes the available energy over active modes. On the other hand, for $\omega \gg \omega_{1}$ the spectral power will follow the power law Eq. (85). Thus, we may write:

$F(\omega) \sim \frac{1}{\omega^{\frac{4}{3}}}, \quad 5 \omega_{1} \lesssim \omega \lesssim 2 m_{\mathrm{e}}$,

where we have somewhat arbitrarily assumed the low energy cutoff $\sim 5 \omega_{1}$. On the other hand, for $\omega \lesssim 5 \omega_{1}$ the redistribution of the energy by resonant synchrotron scattering over electron and proton modes leads to an effective description of the relevant luminosity as a thermal blackbody with effective temperature and radius $T_{2}$ and $R_{2}$. Obviously, the blackbody radius $R_{2}$ is fixed by the geometrical constraint that the radiation is emitted in the magnetosphere at distances $r \lesssim 10 R$, so that we have:

$R_{2} \lesssim 10 R$

The effective blackbody temperature $T_{2}$ can be estimated by observing that the integral of the spectral power up to $5 \omega_{1}$ accounts for about $60 \%$ of the total luminosity. Thus, we have:

$L_{2}(t) \simeq 0.60 L(t)$

where

$L_{2}=4 \pi R_{2}^{2} \sigma_{\mathrm{SB}} T_{2}^{4}, \quad R_{2} \lesssim 10 R$.

Equations (102) and (103) can be used to to determine the effective blackbody temperature. If we again consider the giant 
burst from SGR 1900+14, soon after the hard spike, assuming $R_{2} \simeq 10 R$, we readily obtain:

$T_{2}(0) \simeq 27 \mathrm{keV}$.

To summarize, we have found that the spectral luminosities can be accounted for by two blackbodies and a power law. In particular for the blackbody components, we have:

$R_{1} \lesssim R, \quad R_{2} \lesssim 10 R ; T_{2}<T_{1}, R_{1}<R_{2}$

$L_{1} \simeq 0.15 \mathrm{~L}, \quad L_{2} \simeq 0.60 \mathrm{~L}$.

Interestingly enough, Eq. (105) displays an anticorrelation between blackbody radii and temperatures, in fair agreement with observations. Moreover, the remaining $25 \%$ of the total luminosity is accounted for by a power law leading to the high energy tail of the spectral flux

$\frac{\mathrm{d} N}{\mathrm{~d} E} \sim E^{-\alpha}, \quad \alpha \simeq 2.33$,

extending up to $E \simeq 2 m_{\mathrm{e}} \simeq 1 \mathrm{MeV}$. The high energy power law tail is clearly displayed in the giant flare from SGR 1900+14 (see Fig. 3 in Feroci et al. 1999) and in the recent gigantic flare from SGR 1806-20 (see Fig. 4 in Hurley et al. 2005).

It is customary to fit the spectra with the sum of a power law and an optically thin thermal bremsstrahlung. It should be stressed that the optically thin thermal bremsstrahlung model is purely phenomenological. In view of this, a direct comparison of our proposal with data is problematic. Fortunately, Feroci et al. (2001) tested several spectral functions to the observed spectrum in the afterglow of the giant outburst from SGR 1900+14. In particular they found that, in the time interval $68 \mathrm{~s} \lesssim t \lesssim 195 \mathrm{~s}$, the minimum $\chi^{2}$ spectral model were composed of two blackbody laws plus a power law. By fitting the time-averaged spectra, they reported (Feroci et al. 2001):

$T_{2} \simeq 9.3 \mathrm{keV}, \quad T_{1} \simeq 20.3 \mathrm{keV}, \quad \alpha \simeq 2.8$.

Moreover, it turns out that the power law accounts for approximately $10 \%$ of the total energy above $25 \mathrm{keV}$, while the lowtemperature blackbody component accounts for about $85 \%$. In view of our neglecting the contribution from protons to this energy, we see that our proposal is in accordance with the observed energy balance. Unfortunately, in Feroci et al. (2001) the blackbody radii are not reported. To compare our estimate of the blackbody temperatures with the fitted values in Eq. (107), we note that the values reported in Eqs. (99) and (104) correspond to the blackbody temperatures soon after the initial hard spike. Thus, we need to determine how the blackbody temperatures evolve with time. In Sect. 5 we go on to show that the fading of the luminosity can be accurately reproduced without precise knowledge of the microscopic dissipative mechanisms. In particular, the relevant light curve is given by Eqs. (123) and (127). At $t=0$ the total luminosity is described well by three different spectral components. During the fading of the luminosity, it could happen that microscopic dissipative processes modify the different spectral components. However, it is easy to argue that this does not happens. The crucial point is that the three spectral components originate from emission by a macroscopic part of the magnetosphere, while the time

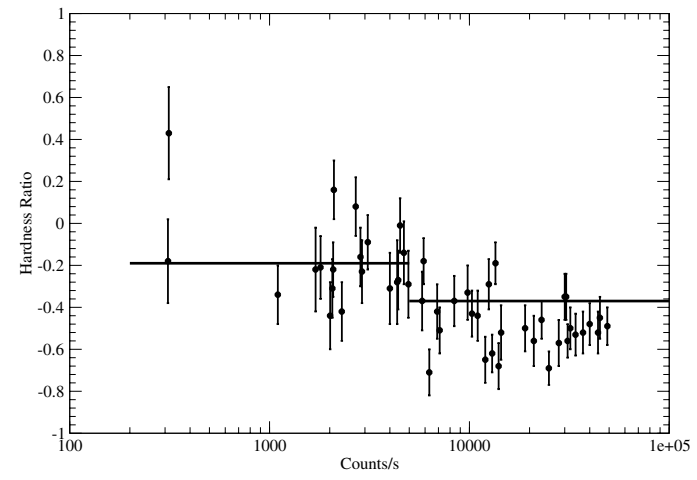

Fig. 4. Hardness-intensity plot of the time-resolved hardness ratio, Eq. (110). Data have been extracted from Fig. 3 in Gotz et al. (2004). Full lines are our Eqs. (115) and (117).

needed to modify a large volume of the magnetosphere by microscopic processes is much longer than the dissipation time $t_{\text {dis }} \sim 10^{2} \mathrm{~s}$. Then we conclude that, even during the fading of the luminosity, the decomposition of the luminosity into three different spectral components retains its validity. Using the results in Sect. 5, we now find:

$\frac{L(t \simeq 68 \mathrm{~s})}{L(0)} \simeq 3.67 \times 10^{-2}, \frac{L(t \simeq 195 \mathrm{~s})}{L(0)} \simeq 1.67 \times 10^{-2}$

Combining Eq. (108) with Eqs. (97), (98), (102), and (103), we obtain

$T_{2}(t \simeq 68 \mathrm{~s}) \simeq 11.8 \mathrm{keV}, T_{2}(t \simeq 195 \mathrm{~s}) \simeq 9.7 \mathrm{keV}$,

$T_{1}(t \simeq 68 \mathrm{~s}) \simeq 26.7 \mathrm{keV}, T_{1}(t \simeq 195 \mathrm{~s}) \simeq 21.9 \mathrm{keV}$,

which is in reasonable agreement with Eq. (107).

Finally, let us comment on the time evolution of the spectral exponent $\alpha$ in the power law Eq. (106). From Eq. (85) it follows that high energy modes have less energy to dissipate. Accordingly, once a finite amount of energy is stored in the magnetosphere, the modes with higher energy become inactive before the lower energy modes. As a consequence, the effective spectral exponent will increase with time and the high energy tail of the emission spectrum becomes softer. This also explains why the fitted spectral exponent $\alpha$ in Eq. (107) is slightly higher than our estimate in Eq. (106).

\subsection{Hardness ratio}

Recently, evidence has been reported of a hardness-intensity anti correlation within bursts from SGR 1806-20 (Gotz et al. 2004). They report observations of the soft gamma ray repeaters SGR 1806-20 obtained in October 2003 during a period of bursting activity. They found that some bursts showed a significant spectral evolution. However, in the present section we focus on the remarkable correlation between the hardness ratio and count rate.

Following Gotz et al. (2004) we define the hardness ratio as:

$H R=\frac{H-S}{H+S}$ 
where $H$ and $S$ are the background subtracted counts in the ranges $40-100 \mathrm{keV}$ and $15-40 \mathrm{keV}$ respectively. Figure 4 reports the hardness ratio data extracted from Fig. 3 in Gotz et al. (2004).

A few comments are in order. First, the hardness ratio becomes negative for sufficiently high burst intensities. Moreover, there is a clear decrease in the hardness ratio with increasing burst intensities. We now show that within our approach we may explain why the hardness ratio is negative and decreases with increasing burst intensities. The hardness ratio Eq. (110) is defined in terms of total luminosities in the relevant spectral intervals. Thus, to determine the total luminosity in the spectral interval $\omega_{1}-\omega_{2}$, we may use

$L\left(\omega_{1}-\omega_{2}\right)=\int_{\omega_{1}}^{\omega_{2}} F(\omega) \mathrm{d} \omega$

where $F(\omega)$ is given by Eq. (85). A straightforward integration gives

$L\left(\omega_{1}-\omega_{2}\right) \simeq 2 \pi^{2} n_{\mathrm{e}} e \frac{\delta B_{\mathrm{S}}}{\delta t_{\text {spike }}} R^{4}\left(\frac{r_{1}}{R}-\frac{r_{2}}{R}\right)$,

where $r_{1}$ and $r_{2}$ are given by:

$\omega_{1,2} \simeq \gamma^{2} \frac{e B_{\mathrm{S}}}{m_{\mathrm{e}}}\left(\frac{R}{r_{1,2}}\right)^{3}$.

Assuming $\gamma \sim 1$, we may rewrite Eq. (113) as:

$\omega_{1,2} \simeq 10 \mathrm{MeV}\left(\frac{R}{r_{1,2}}\right)^{3}$.

Using Eqs. (112) and (114) it is easy to determine the hardness ratio:

$$
\begin{aligned}
H R & =\frac{L(40-100 \mathrm{keV})-L(15-40 \mathrm{keV})}{L(40-100 \mathrm{keV})+L(15-40 \mathrm{keV})} \\
& \simeq \frac{1.66-2.44}{1.66+2.44} \simeq-0.19 .
\end{aligned}
$$

In Fig. 4 we display our estimate of the hardness ratio Eq. (115), and see that the data agree quite closely with Eq. (115) at least up to the count rate $\sim 5 \times 10^{3}$ counts/s. For higher count rates data seem to lie below our value. We believe that, within our approach, there is a natural explanation for this effect. Indeed, for increasing count rates we expect that the hard tail $\omega \gtrsim 2 m_{\mathrm{e}} \simeq 1 \mathrm{MeV}$ of the spectrum will begin to contribute to the luminosity. According to the discussion in Sect. 4.3, these hard photons are reprocessed leading to an effective blackbody with temperature $T_{1}$. Then, for small and intermediate bursts the blackbody temperature $T_{1}$ is considerably lower than Eq. (99), so that the effective blackbody contributes mainly to the soft tail of the spectrum. Obviously, the total luminosity of the effective blackbody is:

$L(1-10 \mathrm{MeV}) \simeq 2 \pi^{2} n_{\mathrm{e}} e \frac{\delta B_{\mathrm{S}}}{\delta t_{\text {spike }}} R^{4}(2.15-1)$.

Since this luminosity contributes to the soft part of the emission spectrum, Eq. (115) gets modified as:

$H R \simeq \frac{1.66-3.59}{1.66+3.59} \simeq-0.37$.
Equation (117) is displayed in Fig. 4 for rates $\gtrsim 5 \times$ $10^{3}$ counts/s. Note that we did not take the proton contribution to the luminosity into account. That protons contribute mainly to luminosities at low energy $\omega \lesssim 10 \mathrm{keV}$ shows that adding the proton contributions lead to lower hardness ratios bringing our estimates in closer agreement with the data. In any case, we see that our theory allows the anti correlation between hardness ratio and intensity to be explained in a natural way.

\section{Light curves}

In our magnetar theory the observed burst activities are triggered by glitches that inject magnetic energy into the magnetosphere where it is dissipated, as discussed in previous sections. As a consequence the observed luminosity is time-dependent. In this section we set up an effective description that allows us to determine the light curves, i.e. the time dependence of the luminosity.

In general, the energy injected into the magnetosphere after the glitch decreases due to the dissipative effects described in Sect. 4.3, leading to the luminosity $L(t)=-\frac{\mathrm{d} E(t)}{\mathrm{d} t}$. Actually, the precise behavior of $L(t)$ is determined once the dissipation mechanisms are known. However, since the dissipation of the magnetic energy involves the whole magnetosphere, we may accurately reproduce the time variation of $L(t)$ without precise knowledge of the microscopic dissipative mechanisms. Indeed, on general grounds we find that the dissipated energy is given by:

$L(t)=-\frac{\mathrm{d} E(t)}{\mathrm{d} t}=\kappa(t) E^{\eta}, \quad \eta \leq 1$,

where $\eta$ is the efficiency coefficient. Obviously parameter $\kappa(t)$ does depend on the physical parameters of the magnetosphere. For an ideal system, where the initial injected energy is huge, the linear regime where $\eta=1$ is appropriate. Moreover, we expect that the dissipation time $\sim \frac{1}{K}$ is much smaller than the characteristic time needed for macroscopic modifications of the magnetosphere. Thus, we may safely assume that $\kappa(t) \simeq \kappa_{0}$ constant. Thus we get:

$L(t)=-\frac{\mathrm{d} E(t)}{\mathrm{d} t} \simeq \kappa_{0} E$

It is then straightforward to solve Eq. (119):

$E(t)=E_{0} \exp \left(-\frac{t}{\tau_{0}}\right), \quad L(t)=L_{0} \exp \left(-\frac{t}{\tau_{0}}\right)$,

$L_{0}=\frac{E_{0}}{\tau_{0}}, \quad \tau_{0}=\frac{1}{\kappa_{0}}$

Note that the dissipation time $\tau_{0}=\frac{1}{\kappa_{0}}$ encodes all the physical information on the microscopic dissipative phenomena. Since the injected energy is finite, the dissipation of energy degrades with the decrease in the available energy. Thus, the relevant equation is Eq. (118) with $\eta<1$. In this case, by solving Eq. (118) we find:

$L(t)=L_{0}\left(1-\frac{t}{t_{\mathrm{dis}}}\right)^{\frac{\eta}{1-\eta}}$ 
where we have introduced the dissipation time:

$t_{\text {dis }}=\frac{1}{\kappa_{0}} \frac{E_{0}^{1-\eta}}{1-\eta}$.

Then, we see that the time evolution of the luminosity is linear up to some time $t_{\text {break }}$, and after that we have a break from the linear regime $\eta=1$ to a non linear regime with $\eta<1$. If we indicate the total dissipation time by $t_{\mathrm{dis}}$, we get:

$L(t)=L_{0} \exp \left(-\frac{t}{\tau_{0}}\right), \quad 0<t<t_{\text {break }}$,

$L(t)=L\left(t_{\text {break }}\right)\left(1-\frac{t-t_{\text {break }}}{t_{\text {dis }}-t_{\text {break }}}\right)^{\frac{\eta}{1-\eta}}, t_{\text {break }}<t<t_{\text {dis }}$.

Equation (123) is relevant for describing the light curve after a giant burst, where there is a huge amount of magnetic energy dissipated into the magnetosphere.

It is interesting to compare our light curves, Eq. (123), with the standard magnetar model. The decay of the luminosity in the standard magnetar model is due to the evaporation by a fireball formed after a giant burst and trapped onto the stellar surface (Duncan \& Thompson 1996, 2001). Indeed, Feroci et al. (2001) considered the light curves after the giant flare of August 27, 1998 from SGR 1900+14, and the giant flare of March 5, 1979 from SGR 0526-66. Assuming that the luminosity varies as a power of the remaining fireball energy $L \sim E^{a}$, they find

$L(t)=L_{0}\left(1-\frac{t}{t_{\text {evap }}}\right)^{\frac{a}{1-a}}$,

where $t_{\text {evap }}$ is the time at which the fireball evaporates, and index $a$ accounts for the geometry and the temperature distribution of the trapped fireball. For a spherical fireball of uniform temperature $a=\frac{2}{3}$, so that the index $a$ must satisfies the constrain:

$a \leqslant \frac{2}{3}$.

Note that our Eq. (123) reduces to Eq. (124), if $t_{\text {break }}=0$ and $\eta=a$. However, we stress that our efficiency exponent must satisfy the milder constraint $\eta \leqslant 1$.

Feroci et al. (2001) perform a best fit of the light curve of the August 27 flare, background subtracted and binned to $5 \mathrm{~s}$, to Eq. (124) and find:

$a=0.756 \pm 0.003, \quad t_{\text {evap }}=414 \mathrm{~s}$.

Indeed, from their Fig. 2 one sees that the trapped fireball light curve accounts for the decay trend of the experimental light curve and matches the sudden final drop in the flux. However, the fitting parameter $a$ in Eq. (126) does not satisfy the physical constraint Eq. (125). Even more, any deviations from spherical geometry or uniform temperature distribution lead to parameters $a$ that are smaller than the upper bound $\frac{2}{3}$. Moreover, the trapped fireball light curve underestimate the measured flux by about an order of magnitude during the first stage of the outburst. We interpreted the different behavior of the flux during the initial phase of the outburst as a clear indication of the linear regime described by our Eq. (120). As a matter of fact,

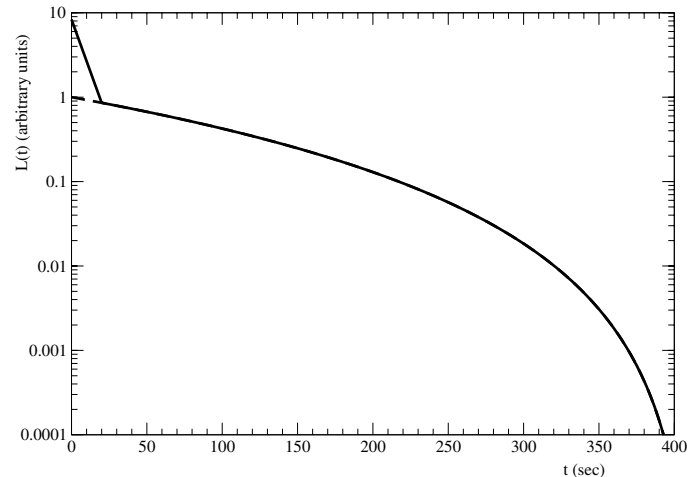

Fig. 5. Light curve after the giant flare of August 27, 1998 from SGR 1900+14. The dashed line is the light curve in the standard magnetar model, Eq. (124), with parameters given in Eq. (126). The full line is our light curve Eq. (123) with the parameters in Eq. (127).

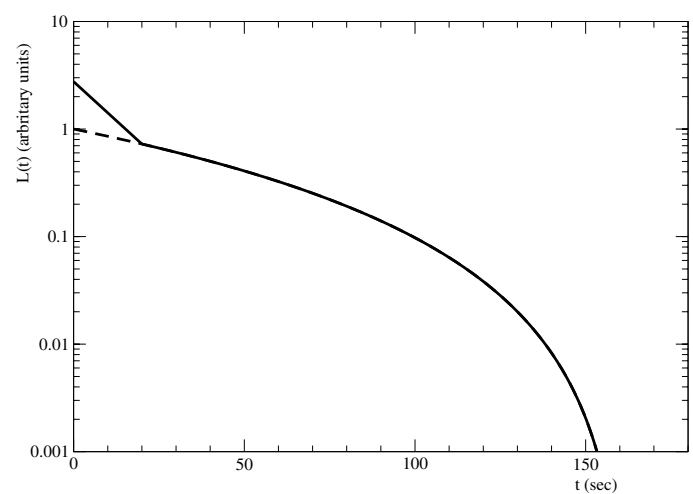

Fig. 6. Light curve after the giant flare of March 5, 1979 from SGR 0526-66. The dashed line is the light curve in the standard magnetar model, Eq. (124), with parameters given in Eq. (128). The full line is our light curve Eq. (123) with the parameters in Eq. (129).

we find that the measured light curve could be better described by Eq. (123) with the parameters (see Fig. 5)

$\tau_{0}=8.80 \mathrm{~s}, \quad t_{\text {break }}=20 \mathrm{~s}$,

$\eta=0.756, \quad t_{\mathrm{dis}}=414 \mathrm{~s}$.

The same criticisms apply to the fit within the standard magnetar model of the light curve after the giant flare of March 5, 1979 from SGR 0526-66. The trapped fireball light curve fit in Feroci et al. (2001) gives:

$a=0.71 \pm 0.01, \quad t_{\text {evap }}=163 \pm 5 \mathrm{~s}$.

Again parameter $a$ exceeds the bound Eq. (125), and the fit underestimates the flux during the first stage of the outburst (see Fig. 14 in Feroci et al. 2001). Fitting our Eq. (123) to the measured flux reported in Fig. 14 in Feroci et al. (2001), we estimate

$\tau_{0}=15 \mathrm{~s}, \quad t_{\text {break }}=20 \mathrm{~s}$,

$\eta=0.71, \quad t_{\text {dis }}=163 \mathrm{~s}$.

In Figs. 5 and 6 we compare our light curves Eqs. (123), (127), and (129) with the best fits performed in Feroci et al. (2001). Obviously, both light curves agree for $t>t_{\text {break }}$, while in the linear regime $t<t_{\text {break }}$, where the trapped fireball light curves 
underestimate the flux, our light curves follow the exponential decay and seem to agree more closely with observational data.

Several observations indicate that after a giant burst there are smaller and more recurrent bursts. According to our theory they are the effect of several small glitches following the giant glitch. We may think about these small bursts as similar to the seismic activity following a giant earthquake. These seismic glitches are characterized by very different light curves from the giant burst light curves.

In the standard magnetar model these light curves are accounted for with an approximate $t^{-0.7}$ decay (Lyubarski et al. 2002). Within our theory there is a natural way to describe the seismic burst activity. Indeed, during these seismic bursts that we shall call settling bursts, there is an almost continuous injection of energy into the magnetosphere, which tends to sustain an almost constant luminosity. This corresponds to an effective $\kappa$ in Eq. (118) which decreases smoothly with time. The simplest choice is:

$\kappa(t)=\frac{\kappa_{0}}{1+\kappa_{1} t}$.

Inserting this into Eq. (118) and integrating, we get:

$E(t)=\left[E_{0}^{1-\eta}-(1-\eta) \frac{\kappa_{0}}{\kappa_{1}} \ln \left(1+\kappa_{1} t\right)\right]^{\frac{\eta}{1-\eta}}$,

so that the luminosity is:

$L(t)=\frac{L_{0}}{\left(1+\kappa_{1} t\right)^{\eta}}\left[1-(1-\eta) \frac{\kappa_{0}}{\kappa_{1} E_{0}^{1-\eta}} \ln \left(1+\kappa_{1} t\right)\right]^{\frac{\eta}{1-\eta}}$.

After defining the dissipation time as

$\ln \left(1+\kappa_{1} t_{\mathrm{dis}}\right)=\frac{\kappa_{1}}{\kappa_{0}} \frac{E_{0}^{1-\eta}}{1-\eta}$,

we rewrite Eq. (132) as

$L(t)=\frac{L_{0}}{\left(1+\kappa_{1} t\right)^{\eta}}\left[1-\frac{\ln \left(1+\kappa_{1} t\right)}{\ln \left(1+\kappa_{1} t_{\mathrm{dis}}\right)}\right]^{\frac{\eta}{1-\eta}}$.

Note that the light curve in Eq. (134) depends on two characteristic time constants $\frac{1}{\kappa_{1}}$ and $t_{\text {dis }}$. We see that $\kappa_{1} t_{\text {dis }}$, which is roughly the number of small bursts that occurred in the given event, gives an estimation of the seismic burst intensity. Moreover, since during the seismic bursts the injected energy is much smaller than in giant bursts, we expect that fitting Eq. (134) to the observed light curves will result in lower values of $\eta$ than is typical values in giant bursts. Below we show that our light curves Eq. (134) are indeed in good agreement with several observations.

\subsection{AXP 1E $2259+586$}

On 2002, June 18 SGR-like bursts were recorded from AXP 1E 2259+586. Coincident with the burst activity were gross changes in the pulsed flux, persistent flux, energy spectrum, pulse profile, and spin-down of the source (Woods et al. 2004). As discussed in previous sections, these features are

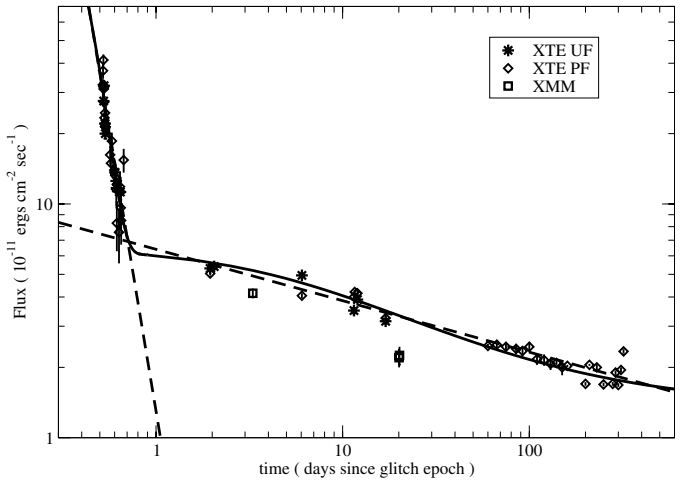

Fig. 7. The time evolution of the unabsorbed flux from AXP 1E 2259+586 following the 2002 June outburst. Data have been extracted from Fig. 13 in Woods et al. (2004). Dashed lines are the phenomelogical power law fits, Eq. (135). The full line is our light curve Eq. (137), with parameters in Eq. (140).

naturally accounted for within our theory. However, we believe that the most remarkable and compelling evidence for our proposal comes from the observed coincidence of the burst activity with a large glitch. Moreover, the time evolution of the unabsorbed flux from AXP 1E 2259+586 following the 2002 June outburst reported in Woods et al. (2004) can be explained naturally within our theory. We could consider the June 18 SGR-like bursts from AXP 1E 2259+586 the Rosetta Stone for our magnetar theory.

The temporal decay of the flux during the burst activity displays a rapid initial decay that lasted about 1 day, followed by a milder decay during the year following the onset of the burst activity. Indeed, Woods et al. (2004) split the data into two segments, and fit each independently to a power law:

$F(t) \sim t^{\alpha_{1}}, \alpha_{1}=-4.8 \pm 0.5, t \lesssim 1$ days,

$F(t) \sim t^{\alpha_{2}}, \alpha_{2}=-0.22 \pm 0.01, t \gtrsim 1$ days.

It is evident from Eq. (135) that the standard magnetar model is completely unable to reproduce the phenomelogical power law fit. On the other hand, even the phenomenological parametrization cannot account for the time evolution of the flux. Indeed, if we assume the power law Eq. (135) for the decay of the flux, then we cannot explain why and how the source returns in its quiescent state with quiescent flux (Woods et al. 2004):

$F_{\mathrm{Q}} \simeq 1.53 \times 10^{-11} \frac{\mathrm{erg}}{\mathrm{cm}^{2} \mathrm{~s}}$.

Note that adding the quiescent flux to the power law decay does not resolve the problem, for in that case the fits worsen considerably. Our interpretation of the puzzling light curve displayed in Fig. 7 is that AXP 1E 2259+586 has undergone a giant burst at the glitch epoch, and soon after the pulsar has entered into intense seismic burst activity. Accordingly, the flux can be written as

$F(t)=F_{\mathrm{GB}}(t)+F_{\mathrm{SB}}(t)+F_{\mathrm{Q}}$

where $F_{\mathrm{Q}}$ is the quiescent flux, Eq. (136); $F_{\mathrm{GB}}(t)$ is the giant burst contribution to the flux given by Eq. (123); and $F_{\mathrm{SB}}(t)$ is the seismic burst contribution given by Eq. (134). Since there 
are no available data during the first stage of the outburst, we may parameterize the giant burst's contribution as

$F_{\mathrm{GB}}(t)=F_{\mathrm{GB}}(0)\left(1-\frac{t}{t_{\mathrm{GB}}}\right)^{\frac{\eta_{\mathrm{GB}}}{1-\eta_{\mathrm{GB}}}}, \quad 0<t<t_{\mathrm{GB}}$,

while $F_{\mathrm{SB}}(t)$ is given by:

$F_{\mathrm{SB}}(t)=\frac{F_{\mathrm{SB}}(0)}{\left(1+\kappa_{1} t\right)^{\eta_{\mathrm{SB}}}}\left[1-\frac{\ln \left(1+\kappa_{1} t\right)}{\ln \left(1+\kappa_{1} t_{\mathrm{SB}}\right)}\right]^{\frac{\eta_{\mathrm{SB}}}{1-\eta_{\mathrm{SB}}}}, 0<t<t_{\mathrm{SB}}$

where $t_{\mathrm{GB}}$ and $t_{\mathrm{SB}}$ are the dissipation time for giant and seismic bursts, respectively. In Fig. 7 we display our light curve Eq. (137) with the following parameters:

$$
\begin{aligned}
& F_{\mathrm{GB}}(0) \simeq 1.5 \times 10^{-8} \frac{\mathrm{erg}}{\mathrm{cm}^{2} \mathrm{~s}} \\
& \eta_{\mathrm{GB}} \simeq 0.828, t_{\mathrm{GB}} \simeq 0.91 \text { days } \\
& F_{\mathrm{SB}}(0) \simeq 5.0 \times 10^{-11} \frac{\mathrm{erg}}{\mathrm{cm}^{2} \mathrm{~s}} \\
& \eta_{\mathrm{SB}} \simeq 0.45, t_{\mathrm{SB}} \simeq 10^{3} \text { days, } \kappa_{1} \simeq 0.20 \text { days }^{-1} .
\end{aligned}
$$

A few comments are in order. First, the agreement with data is rather good. Second, our efficiency exponent $\eta_{\mathrm{GB}}$ is consistent with the values found in the giant bursts from SGR 1900+14 and SGR 0526-66. On the other hand, we have $\eta_{\mathrm{SB}}<\eta_{\mathrm{GB}}$ quite consistently. Finally, it follows from our interpretation of the light curve that the onset of the intense seismic burst activity $\left(\kappa_{1} t_{\mathrm{SB}} \sim 200\right)$ did not allow a reliable estimation of $\frac{\delta \dot{v}}{\dot{v}}$, which we predicted to be on the order of $10^{-2}$.

Interestingly enough, following the 2002 June outburst an infrared flux change was detected that correlated with the X-ray flux variability (Tam et al. 2004). Since the observations began three days after the 2002 June outburst, according to our theory the infrared flux is parameterized as:

$$
\begin{aligned}
F_{\mathrm{SB}}^{\mathrm{IR}}(t)= & \frac{F_{\mathrm{SB}}^{\mathrm{IR}}(0)}{\left(1+\kappa_{1} t\right)^{\eta_{\mathrm{SB}}}}\left[1-\frac{\ln \left(1+\kappa_{1} t\right)}{\ln \left(1+\kappa_{1} t_{\mathrm{SB}}\right)}\right]^{\frac{\eta_{\mathrm{SB}}}{1-\eta_{\mathrm{SB}}}} \\
& +F_{\mathrm{Q}}^{\mathrm{IR}}, \quad 0<t<t_{\mathrm{SB}},
\end{aligned}
$$

with the same parameters as in Eq. (139). Indeed, assuming $F_{\mathrm{SB}}^{\mathrm{IR}}(0) \simeq 9.5 \times 10^{-15} \frac{\mathrm{erg}}{\mathrm{cm}^{-2} \mathrm{~s}^{-1}}$ and $F_{\mathrm{Q}}^{\mathrm{IR}} \simeq 3.3 \times 10^{-15} \frac{\mathrm{erg}}{\mathrm{cm}^{-2} \mathrm{~s}^{-1}}$, we found that our light curve Eq. (141) is in remarkably good agreement with the data (see Fig. 8). The strong correlation between the infrared and X-ray flux decays observed after the 2002 June outburst from AXP 1E 2259+586 strongly suggests a physical link between the origins of both types of radiation.

\subsection{SGR $1900+14$}

Soon after the August 27, 1998 giant burst, the soft gamma repeater SGR 1900+14 entered a remarkable phase of activity. On August 29 an unusual burst from SGR 1900+14 was detected (Ibrahim et al. 2001), which lasted for a long time $\sim 10^{3} \mathrm{~s}$. As discussed in Ibrahim et al. (2001), on observational grounds extended afterglow tails following ordinary bursts can be ruled out.

In Fig. 9 we display the flux decay after the August 29 burst. Data has been extracted from Ibrahim et al. (2001), where the

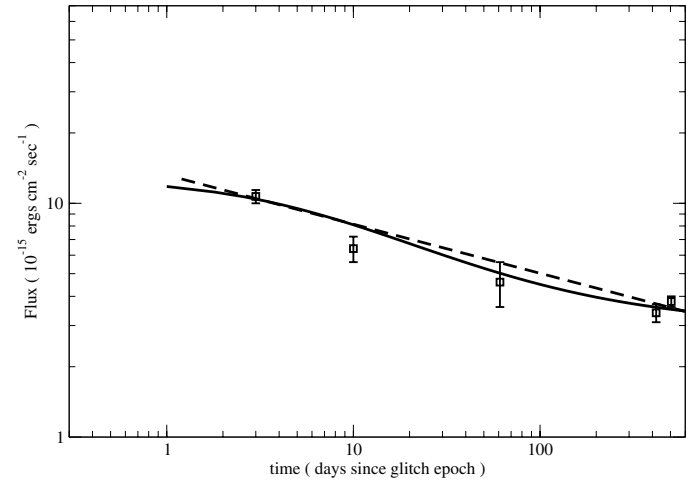

Fig. 8. The time evolution of the unabsorbed IR flux from AXP 1E 2259+586 following the 2002 June outburst. Data have been extracted from Fig. 1 in Tam et al. (2004). The dashed line is the phenomelogical power law fit $t^{-0.21 \pm 0.01}$. The full line is our light curve Eq. (141).

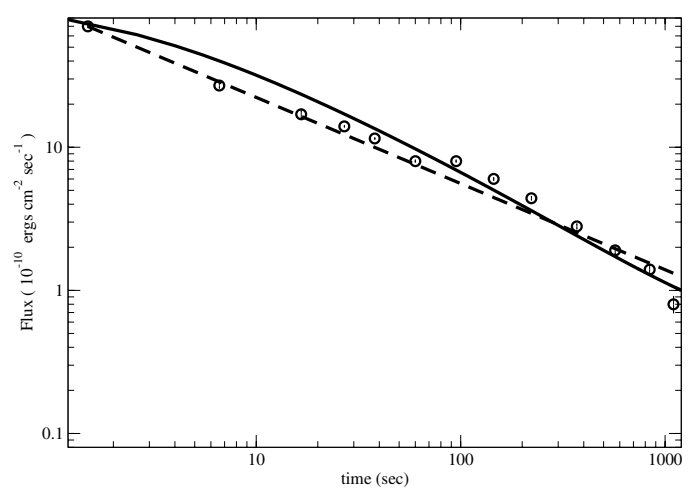

Fig. 9. Flux evolution of the August 29 burst from SGR 1900+14. Data has been extracted from Fig. 4, panel (d), in Ibrahim et al. (2001). The dashed line is the phenomenological power law fit Eq. (142); the full line is our light curve Eqs. (144) and (145).

temporal behavior of the flux decay has been parameterized as a power law (dashed line in Fig. 9):

$F(t)=(89.16 \pm 1.34) \times 10^{-10} \frac{\mathrm{erg}}{\mathrm{cm}^{2} \mathrm{~s}} t^{-(0.602 \pm 0.025)}$.

As already stressed, the phenomenological power law decay cannot explain the return of the source in its quiescent state where the flux is (Hurley et al. 1999):

$F_{\mathrm{Q}}=0.96 \pm 0.07 \times 10^{-11} \frac{\mathrm{erg}}{\mathrm{cm}^{2} \mathrm{~s}}$.

On the other hand, we may easily account for the observed flux decay by our light curve:

$F(t)=\frac{F(0)}{\left(1+\kappa_{1} t\right)^{\eta}}\left[1-\frac{\ln \left(1+\kappa_{1} t\right)}{\ln \left(1+\kappa_{1} t_{\mathrm{dis}}\right)}\right]^{\frac{\eta}{1-\eta}}+F_{\mathrm{Q}}$,

where $F_{\mathrm{Q}}$ is fixed by Eq. (143). Indeed, in Fig. 9 we compare our light curve Eq. (144) with observational data. The agreement is quite satisfying, if we take:

$F(0) \simeq 1.05 \times 10^{-9} \frac{\mathrm{erg}}{\mathrm{cm}^{2} \mathrm{~s}}, \eta \simeq 0.5$,

$t_{\text {dis }} \simeq 1.2 \times 10^{3} \mathrm{~s}, \kappa_{1} \simeq 0.50 \mathrm{~s}^{-1}$. 


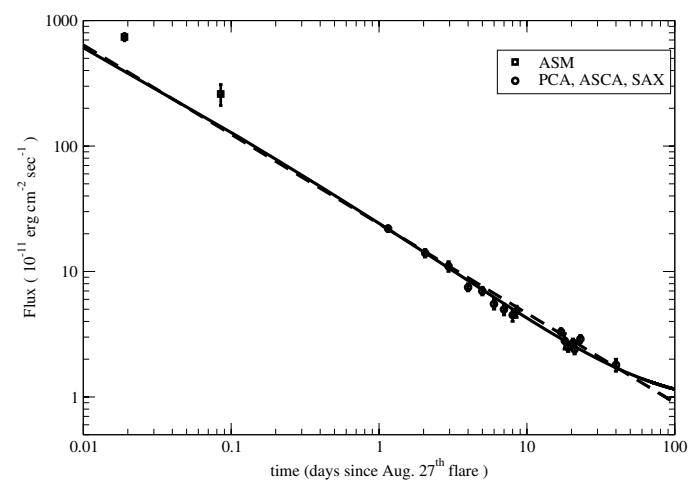

Fig. 10. The time evolution of the unabsorbed flux from SGR 1900+14 following the 1998 August outburst. Data has been extracted from Fig. 2 in Woods et al. (2001). The dashed line is the power law best fit $F(t) \sim t^{-(0.713 \pm 0.025)}$. The full line is our light curve Eqs. (144) and (146).

Woods et al. (2001) have analyzed a large set of X-ray observations of SGR 1900+14 in order to construct a more complete flux history. They found that the flux level was more than an order of magnitude brighter than the level during quiescence. This transient flux enhancement lasts about 40 days after the giant flare. Unlike Woods et al. (2001), which argued that this enhancement was an artifact of the August 27 flare, we believe that the flux history can be adequately described as seismic burst activity of the source. In Fig. 10 we report the flux light curve extracted from Fig. 2 in Woods et al. (2001), together with their power law best fit. Again we find that the flux history is accounted for quite well by our light curve, Eq. (144), with the following parameters:

$F(0) \simeq 4.8 \times 10^{-8} \frac{\mathrm{erg}}{\mathrm{cm}^{2} \mathrm{~s}}, \eta \simeq 0.55$,
$t_{\text {dis }} \simeq 200$ days, $\kappa_{1} \simeq 2 \times 10^{3}$ days $^{-1}$.

The agreement between our light curve in Eqs. (144) and (146) with the power-law best fit is striking. Moreover, we see that our curve deviates from the power law fit for $t>60$ days tending to $F_{\mathrm{Q}}$ at $t=t_{\text {dis }}$.

Woods et al. (2001) noted that by extrapolating the fit to the August 27 X-ray light curve back toward the flare itself, one finds that the expected flux level lies below the ASM flux measurements (squares in Fig. 10). Moreover, these authors observed that the discrepancy reduces somewhat when one pushes the reference epoch forward to about 14 min after the onset of the flare. We believe that this discrepancy is due to a true physical effect, namely the observed discrepancy between extrapolated light curve and ASM measurements is a clear indication that the surface luminosity increases after the burst activity. In particular, soon after the August 27 giant flare, the surface temperature increases up to $\sim 61 \mathrm{keV}$ and the surface luminosity reaches $\sim 10^{44} \frac{\mathrm{erg}}{\mathrm{s}}$ (Sect. 4.3). Almost all the deposited energy is dissipated within the dissipation time of the giant flare $\sim 400 \mathrm{~s}$. Nevertheless, it is natural to expect a more gradual afterglow where a small fraction of the energy deposited onto the star surface is gradually dissipated. As a matter of fact, we find that the observed level of luminosity $L_{\mathrm{X}} \sim 10^{38} \frac{\mathrm{erg}}{\mathrm{s}}$ (assuming a

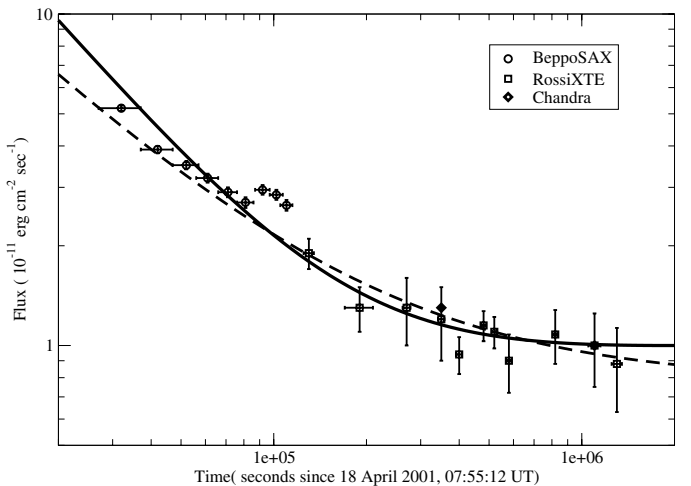

Fig. 11. Temporal behavior of the X-ray flux from SGR $1900+14$ in the aftermath of the April 18, 2001 flare. Data have been extracted from Fig. 2 in Feroci et al. (2003). The dashed line is the power law best fit Eqs. (147) and (148). The full line is our light curve Eqs. (144) and (149).

distance $d=10 \mathrm{kpc}$ ) at about 0.01 days since the August 27 giant flare is consistent with the gradual afterglow scenario.

On April 18, 2001, the soft gamma ray repeater SGR 1900+14 emitted an intermediate burst. The light curve of this event did not show any initial hard spike and was clearly spin-modulated. Moreover, the energetics appeared to be intermediate in the 40-700 keV range, with a total emitted energy of about $1.9 \times 10^{42} \mathrm{erg}$ (Feroci et al. 2003). In Fig. 11 we report the temporal behavior of the X-ray $(2-10 \mathrm{keV})$ flux from SGR 1900+14 in the aftermath of the April 18, 2001 flare. Data has been extracted from Fig. 2 in Feroci et al. (2003), who attempt a simple power law function to the flux data:

$F(t) \sim t^{-\alpha}+K$,

where the constant $K$ should take care of the quiescent luminosity. Indeed, fitting Eq. (147) to the data, Feroci et al. (2003) found:

$\alpha=0.89(6), \quad K=0.78(5) \times 10^{-11} \frac{\mathrm{erg}}{\mathrm{cm}^{2} \mathrm{~s}}$.

As is evident from Fig. 11, the power law fits the data quite nicely. However, the reduced $\chi^{2}$ turns out to be in excess of 3 , mainly due to the bump occurring in the light curve at $t \sim 10^{5} \mathrm{~s}$ (Feroci et al. 2003). After excluding the bump they do get a good fit with $\chi^{2} /$ d.o.f. $\simeq 1$ without any appreciable variation in the fit parameters. However, there is still a problem with the phenomelogical power-law decay of the flux. As a matter of fact, Eq. (148) shows that the power-law fit underestimates the quiescent luminosity. In our opinion this confirms that the phenomenological power-law decay of the flux is not adeguate for describing the time variation of the flux. On the other hand, we find that our light curve in Eq. (144), with quiescent luminosity fixed to the observed value in Eq. (143), furnishes a rather good description of the flux decay once the parameters are given by:

$F(0) \simeq 2.6 \times 10^{-7} \frac{\mathrm{erg}}{\mathrm{cm}^{2} \mathrm{~s}}, \eta \simeq 0.68$

$t_{\mathrm{dis}} \simeq 3 \times 10^{6} \mathrm{~s}, \kappa_{1} \simeq 0.25 \mathrm{~s}^{-1}$.

Within our interpretation, Eq. (149) shows that the flux decay in the aftermath of the April 18 flare is characterized by 


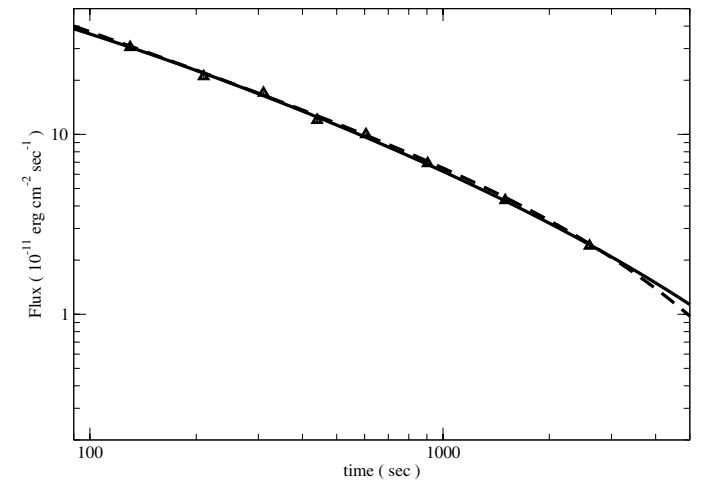

Fig. 12. The temporal decay of the flux from the April 28, 2001 burst from SGR $1900+14$ in the energy band $2-20 \mathrm{keV}$. The data was extracted from Fig. 5 in Lenters et al. (2003). The dashed line is the phenomenological best-fit power law times exponential function adopted in Lenters et al. (2003) to describe data, Eqs. (150) and (151). Full continuous line is our light curve in Eqs. (144) and (152).

a very large seismic burst activity $\left(\kappa_{1} t_{\text {dis }} \sim 10^{6}\right)$, which lasts for about $10^{6} \mathrm{~s}$. As a result, the bump in the flux at $t \sim 10^{5} \mathrm{~s}$ is naturally explained as fluctuations in the intensity of the seismic bursts.

Lenters et al. (2003) report the spectral evolution and temporal decay of the X-ray tail of a burst from SGR 1900+14 recorded on April 28, 2001, 10 days after the intense April 18 event. In Fig. 12 we display the temporal decay of the flux from the April 28, 2001 burst in the energy band 2-20 keV. The data has been extracted from Fig. 5 in Lenters et al. These authors attempted several functional forms to fit the decay of the flux. They reported that the decay was equally well-fitted by either a power law times exponential or a broken power law. We stress that both fits are phenomenological parametrization of the observational data, and that both fits are unable to recover the quiescent flux. For definitiveness, we shall compare our light curve with the power law times exponential fit:

$F(t) \sim t^{-\alpha} \exp \left(-\frac{t}{\tau}\right)$

The best fit to the temporal decay of the flux from the April 28, 2001 burst in the energy band 2-20 keV gives (Lenters et al. 2003):

$\alpha=0.68 \pm 0.04, \quad \tau=5 \pm 1 \times 10^{3} \mathrm{~s}$.

In Fig. 12 we compare the phenomenological best fit Eqs. (150) and (151) with our light curve Eq. (144), where the quiescent luminosity is fixed to the observed value Eq. (143), and the parameters are given by:

$F(0) \simeq 1.4 \times 10^{-9} \frac{\mathrm{erg}}{\mathrm{cm}^{2} \mathrm{~s}}, \eta \simeq 0.5$,

$t_{\text {dis }} \simeq 5.5 \times 10^{3} \mathrm{~s}, \kappa_{1} \simeq 0.06 \mathrm{~s}^{-1}$.

Again, we see that our light curve gives a quite satisfying description of the flux decay.

Let us consider, finally, the light curve for the intermediate burst from SGR 1900+14 that occurred on July 2, 2001 (Olive et al. 2004). Figure 13 displays the time decay of the flux after the July 2 burst. The data were extracted from Fig. 1 in

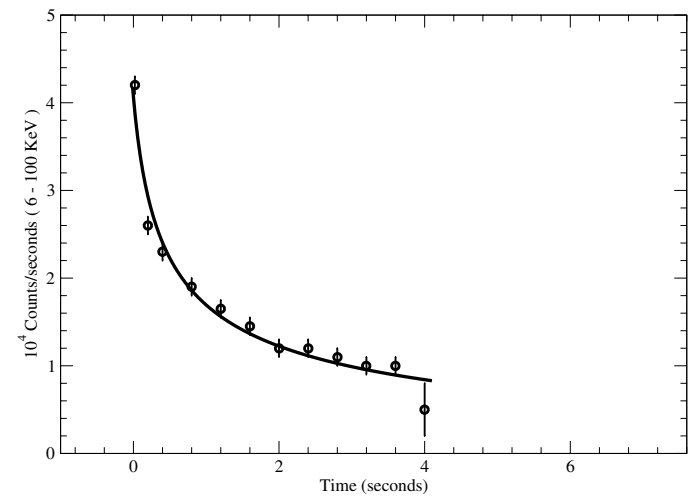

Fig. 13. Time history of the July 2, 2001 burst from SGR 1900+14 in the energy band 7-100 keV as observed by FREGATE. The data was extracted from Fig. 1 in Olive et al. (2004) by binning the light curve histogram. The full line is our light curve Eqs. (144) and (153).

Olive et al. (2004) by binning the light curve histogram. The displayed errors are our estimate, so that the data should be considered as purely indicative of the decay of the flux. We find that Fig. 1 in Olive et al. (2004) is very suggestive, for it seems to indicate that the burst results from several small bursts; i.e. according to our theory the burst is a seismic burst. As a consequence we try the fit with our light curve Eq. (144). In this case the quiescent flux has been fixed to $F_{\mathrm{Q}} \simeq 0$, for the observational data was taken in the energy range $7-100 \mathrm{keV}$ where the quiescent flux is very small. Attempting the fit to the data we find:

$F(0) \simeq 4.07 \times 10^{4} \frac{\text { counts }}{\mathrm{s}}, \eta \simeq 0.36$,

$t_{\mathrm{dis}} \simeq 40 \mathrm{~s}, \kappa_{1} \simeq 5.0 \mathrm{~s}^{-1}$.

The resulting light curve is displayed in Fig. 13. The peculiarity of this burst resides in the fact that the burst activity terminates suddenly at $t \simeq 4 \mathrm{~s}$, well before the natural end at $t_{\mathrm{dis}} \simeq 40 \mathrm{~s}$.

\subsection{SGR $1627-41$}

SGR 1627-41 was discovered with the burst and transient source experiment (BATSE) at the Compton Gamma-Ray Observatory (CGRO) in June 1998 (Kouveliotou et al. 1998; Woods et al. 1999a) when it emitted over 100 bursts within an interval of 6 weeks. Kouveliotou et al. (2003) present the results of the monitoring of the flux decay of the X-ray counterpart of SGR 1627-41 spanning an interval of roughly five years. Moreover, these authors attempted to understand the three year monotonic decline of SGR 1627-41 within the standard magnetar model as cooling after a single deep-crustal heating event coincided with the burst activity in 1998. They assumed an initial energy injection to the crust on the order of $10^{44} \mathrm{erg}$. However, it must be pointed out that this assumption is highly unrealistic, for the estimate of the total energy released in bursts during the activation of SGR 1627-41 ranges between $4 \times$ $10^{42}-2 \times 10^{43} \mathrm{erg}$. In addition, since gamma rays were not detected, they assumed that the conversion efficiency of the total energy released during the activation into soft gamma rays were considerably less than $100 \%$. They also assumed that 


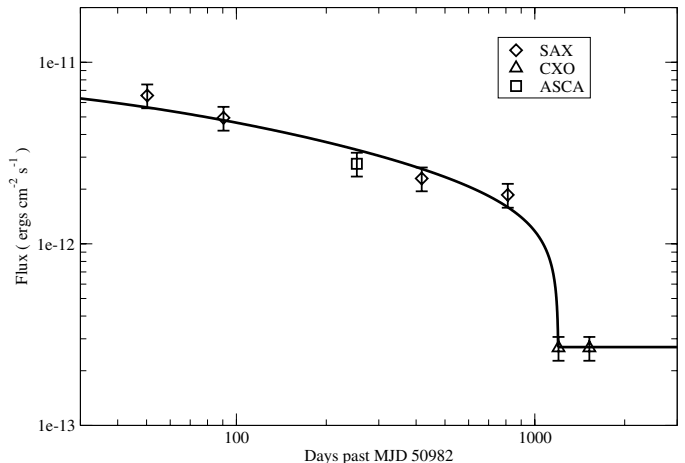

Fig. 14. Time decay of the flux from SGR 1627-41. The data was taken from Table 1 in Kouveliotou et al. (2003). The full line is our bestfitted light curve Eqs. (144), (154), and (155).

the core temperature is low, i.e. the core cools via the direct URCA process. Notwithstanding these rather ad hoc assumptions, Kouveliotou et al. (2003) are unable to explain the March 2003 data point, which clearly shows that the flux did not decay further (see Fig. 14). In other words, the levelling of the flux during the third year, followed by its sharp decline, is a feature that is challenging the standard magnetar model based on neutron stars, which begs for an explanation within that model.

On the other hand, we now show that the peculiar SGR 1627-41 light curve finds a natural interpretation within our theory. In Fig. 14 we display the time decay of the flux. The data was taken from Table 1 in Kouveliotou et al. (2003). In this case we are able to best fit our light curve Eq. (144) to the available data. Since the number of observations is rather low, to get a sensible fit we fixed the dissipation time to 1200 days and the quiescent luminosity to the levelling value at $t \gtrsim 1200$ days:

$F_{\mathrm{Q}} \simeq 2.7 \times 10^{-13} \frac{\mathrm{erg}}{\mathrm{cm}^{2} \mathrm{~s}}, \quad t_{\text {dis }} \simeq 1200$ days.

The best fit of our light curve to data gives:

$F(0)=0.83(11) \times 10^{-11} \frac{\mathrm{erg}}{\mathrm{cm}^{2} \mathrm{~s}}, \eta=0.25(8)$,

$\kappa_{1}=0.04(1)$ days $^{-1}$,

with a reduced $\chi^{2} \sim 1$. From Fig. 14, where we compare our best-fitted light curve with data, we see that our theory allows quite a satisfying description of the three-year monotonic decline of the flux.

\subsection{SGR $1806-20$}

When SGR 1806-20 entered an active phase in 2003 that culminated in a gigantic flare on 2004 December 27,the energy greatly exceeded that of all previous events. Figure 3 in Hurley et al. (2005) displays the time history of the flux averaged over the rotation period of the pulsar soon after the giant flare. These authors fitted the light curve within the standard magnetar model based on the evaporation of a fireball formed after the giant burst and trapped onto the stellar surface, Eq. (124). The fit of the rotation-smoothed curve to the fireball function gives (Hurley et al. 2005):

$a=0.606 \pm 0.003, \quad t_{\text {evap }}=382 \pm 3 \mathrm{~s}$.

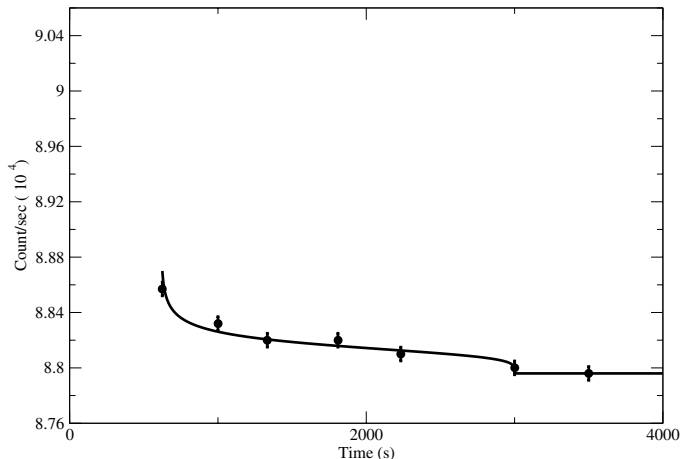

Fig. 15. Time history of the second component after the December 27, 2004 giant burst from SGR 1806-20. The data was extracted from Fig. 5 in Mereghetti et al. (2005b) by binning the light curve histogram. The continuous curve is our light curve Eqs. (144), (157), and (158).

However, from their Fig. 3 it is evident that the fireball light curve underestimates the luminosity for $t \lesssim 30 \mathrm{~s}$. Thus we see that the light curve can be better accounted for by our light curve Eq. (123) with $t_{\text {break }} \simeq 30 \mathrm{~s}$, quite close to the values found for the giant bursts from SGR 1900+14 and SGR 0526-66. A more precise determination of the parameters of our light curve, however, must await more precise data in the initial phase of the afterglow. Instead, in this section we discuss the light curve of a second, separate component after the giant burst reported in Mereghetti et al. (2005b). They find evidence for a separate component in the light curve starting at $t \sim 400 \mathrm{~s}$ from the onset of the giant burst, forming a peak at $t \sim 600 \mathrm{~s}$ and ending at $t \sim 3000 \mathrm{~s}$ (see Fig. 5 in Mereghetti et al. 2005b). As already discussed, our theory expects that there is an intense seismic burst activity following a giant burst. In Fig. 15 we display the flux history starting from the giant flare. We show a few points of the second component extracted from Fig. 5 in Mereghetti et al. (2005b) by binning the light curve histogram. The displayed errors are our estimate, so that the data should be considered as purely indicative of the decay of the flux. We fit the data with our light curve Eqs. (144) assuming:

$F_{\mathrm{Q}} \simeq 8.796 \times 10^{4} \frac{\mathrm{count}}{\mathrm{s}}, \quad t_{\text {dis }}=t_{\text {end }}-t_{\text {start }}$,
$t_{\text {start }} \simeq 625 \mathrm{~s}, \quad t_{\text {end }} \simeq 3000 \mathrm{~s}$.

The best fit of our light curve to data gives:

$F(0)=0.074 \times 10^{4} \frac{\mathrm{count}}{\mathrm{s}}, \eta=0.18, \kappa_{1}=0.10 \mathrm{~s}^{-1}$.

In Fig. 15 we compare our light curve with the data and find that our theory allows a satisfying description of the time history of the flux.

\section{Conclusions}

Let us summarize the main results of the present paper. We have discussed p-stars endowed with super strong dipolar magnetic field and found a well-defined criterion to distinguish rotation-powered pulsars from magnetic powered pulsars (magnetars). We showed that glitches, which in our magnetars 
are triggered by magnetic dissipative effects in the inner core, explain both the quiescent emission and bursts from soft gamma-ray repeaters and anomalous X-ray pulsars. In particular, we were able to account for the braking glitch from SGR 1900+14 and the normal glitch from AXP 1E 2259+586 following a giant burst. We accounted for the observed anti correlation between the hardness ratio and intensity. Within our magnetar theory we were able to account for light curves quantitatively for both gamma-ray repeaters and anomalous X-ray pulsars. In particular we explained the light curve after the June 18, 2002 giant burst from AXP 1E 2259+586. The ability of our p-star theory to lead to a satisfying understanding of several observational features of soft gamma-ray repeaters and anomalous X-ray pulsars supports our proposal of a revision of the standard paradigm of relativistic astrophysics.

Let us conclude by briefly addressing the theoretical foundation of our theory. As a matter of fact, our proposal for p-stars stems from recent numerical lattice results in QCD (Cea \& Cosmai 2003), which suggested that the gauge system gets deconfined in chromomagnetic field that is strong enough. This leads us to consider the new class of compact quark stars made of almost massless, deconfined up-and-down quarks immersed in a chromomagnetic field in $\beta$-equilibrium. Our previous studies showed that these compact stars are stabler than neutron stars whatever the value of the chromomagnetic condensate. This remarkable result indicates that the true ground state of QCD in a sufficiently strong gravitational field is not realized by hadronic matter, but by p-matter. In other words, the final collapse of an evolved massive star inevitably leads to the formation of a p-star.

\section{References}

Alcock, C., Farhi, E., \& Olinto, A. V. 1986, ApJ, 310, 261

Baade, W., \& Zwicky, F. 1934a, Proc. Nat. Acad. Sci., 20, 254

Baade, W., \& Zwicky, F. 1934b, Phys. Rev., 45, 138

Baade, W., \& Zwicky, F. 1934c, Phys. Rev., 46, 76

Baring, M. G., \& Harding, A. K. 1998, ApJ, 507, L55

Burwitz, V., Haberl, F., Neuhäuser, R., et al. 2003, A\&A, 399, 1109

Cameron, P. B., Chandra, P., Ray, A., et al. 2005 [arXiv:astro-ph/0502428]

Cea, P. 2004a, Int. J. Mod. Phys. D, 13, 1917

Cea, P. 2004b, JCAP, 0403011

Cea, P., \& Cosmai, L. 2003, JHEP02, 031

Cheng, B., Epstein, R. I., Guyer, R. A., \& Cody Young, A. 1995, Nature, 382,518

Duncan, R. C., \& Thompson, C. 1992, ApJ, 392, L9

Duncan, R. C., \& Thompson, C. 1993, ApJ, 408, 194

Duncan, R. C., \& Thompson, C. 1995, MNRAS, 275, 255

Duncan, R. C., \& Thompson, C. 1996, ApJ, 473, 322

Duncan, R. C., \& Thompson, C. 2001, ApJ, 561, 980

Feroci, M., Frontera, F., Costa, E., et al. 1999, ApJ, 515, L9

Feroci, M., Hurley, K., Duncan, R. C., \& Thompson, C. 2001, ApJ, 549,1021

Feroci, M., Mereghetti, S., Woods, P., et al. 2003, ApJ, 596, 470

Frail, D. A., Kulkarni, S. R., \& Bloom, J. S. 1999, Nature, 398, 127

Gaensler, B. M., Kouveliotou, C., Gelfand, J. D., et al. 2005, Nature, 434,1104
Gelfand, J. D., Lyubarsky, Y. E., Eichler, D., et al. 2005, ApJ, 634, L89

Ginzburg, V. L. 1979, Theoretical Physics and Astrophysics (Oxford: Pergamon Press)

Gold, T. 1968, Nature, 218, 731

Götz, D., Mereghetti, S., Mirabel, I. F., \& Hurley, K. 2004, A\&A, 417, L45

Granot, J., Ramirez-Ruiz, E., Taylor, G. B., et al. 2006, ApJ, 638, 391 Gundmundsson, E. H., Pethick, C. J., \& Epstein, R. I. 1983, ApJ, 272, 286

Hewish, A., Bell, S. G., Pilkington, et al. 1968, Nature, 217, 709

Hobbs, G., Faulkner, A., Stairs, I. H., et al. 2004, MNRAS, 352, 1439

Hurley, K. 1999 [arXiv: astro-ph/9912061]

Hurley, K. J., McBreen, B., Rabbette, M., \& Steel, S. 1994, A\&A, 288, L49

Hurley, K., Li, P., Kouveliotou, C., et al. 1999, ApJ, 510, L111

Hurley, K., Boggs, S. E., Smith, D. M., et al. 2005, Nature, 434, 1098

Ibrahim, A. I., Strohmayer, T. E., Woods, P. M., et al. 2001, ApJ, 558, 237

Kaspi, V. M. 2004 [arXiv: astro-ph/0402175]

Kaspi, V. M., \& Gavriil, F. P. 2004, Nucl. Phys. Proc. Suppl., 132, 456

Kouveliotou, C., Kippen, M., Woods, et al. 1998, IAU Circ., 6944

Kouveliotou, C., Eichler, D., Woods, P. M., et al. 2003, ApJ, 596, L79

Lenters, G. T., Woods, P. M., Goupell, J. E., et al. 2003, ApJ, 587, 761

Lyubarski, Y., Eichler, D., \& Thompson, C. 2002, ApJ, 580, L69

Manchester, R. N., \& Taylor, J. H. 1997, Pulsars (San Francisco: Freeman, W.H. \& Company)

Marsden, D., \& White, N. E. 2001, ApJ, 551, L155

McLaughlin, M. A., Stairs, I. H., Kaspi, V. M., et al. 2003, ApJ, 591, L135

Mereghetti, S. 1999 [arXiv: astro-ph/9911252]

Mereghetti, S., Chiarlone, L., Israel, G. L., \& Stella, L. 2002 [arXiv: astro-ph/0205122]

Mereghetti, S., Tiengo, A., Esposito, P., et al. 2005a, ApJ, 628, 938

Mereghetti, S., Gotz, D., von Kienlin, A., et al. 2005b, ApJ, 624, L105

Morii, M., Sato, R., Kataoka, J., \& Kawai, N. 2003, PASJ, 55, L45

Nayakshin, S. 2004 [arXiv: astro-ph/0410455]

Olive, J. F., Hurley, K., Sakamoto, T., et al. 2004, ApJ, 616, 1148

Özel, F. 2002, ApJ, 575, 397

Pacini, F. 1968, Nature, 219, 145

Paczyński, B. 1992, Acta Astron., 42, 145

Peebles, P. J. E. 1993, Principles of Physical Cosmology (Princeton: Princeton Univ. Press)

Tam, C. R., Kaspi, V. M., van Kerkwijk, M. H., \& Durant, M. 2004, ApJ, 617, L53

Thompson, C., Duncan, R. C., Woods, P. M., et al. 2000, ApJ, 543, 340

Van Riper, K. A. 1991, ApJ, 372, 251

Wallace, W. H. 1977, Radiation Processes in Astrophysics (Cambridge: MIT Press)

Wang, X. Y., Wu, X. F., Fan, Y. Z., Dai, Z. G., \& Zhang, B. 2005, ApJ, 623, L29

Woods, P. M. 2003 [arXiv: astro-ph/0304372]

Woods, P. M., \& Thompson, C. 2004 [arXiv: astro-ph/0406133]

Woods, P. M., Kouveliotou, C., van Paradijs, J., et al. 1999a, ApJ, 519, L139

Woods, P. M., Kouveliotou, C., van Paradijs, J., et al. 1999b, ApJ, 524, L55

Woods, P. M., Kouveliotou, C., Gögüs, E., et al. 2001, ApJ, 552, 748

Woods, P. M., Kaspi, V. M., Thompson, C., et al. 2004, ApJ, 605, 378 http://www.atnf.csiro.au/research/pulsar/psrcat 